Alloys and Compounds

Elsevier Editorial System(tm) for Journal of

Manuscript Draft

Manuscript Number: JALCOM-D-19-05063R2

Title: Conventional and inverse barocaloric effects in ferroelectric $\mathrm{NH} 4 \mathrm{HSO} 4$

Article Type: Full Length Article

Keywords: Polymorphic phase transformation, Phase diagram, Order-disorder phenomena, Entropy, Barocaloric effect

Corresponding Author: Professor Igor Flerov,

Corresponding Author's Institution: Kirensky Institute of Physics

First Author: Igor Flerov

Order of Authors: Igor Flerov; Mikhail Gorev; Ekaterina Mikhaleva; Evgeniy Bogdanov

Abstract: In this study, the conventional and inverse barocaloric effects (BCE) in ferroelectric NH4HSO4 are reported. Maximum extensive and intensive BCE near order-disorder phase transition can be achieved at low pressure $\mathrm{p} \leq 0.1 \mathrm{GPa}$. Large thermal expansion of the crystal lattice plays a very important role in the developing conventional BCE and conversation between $\mathrm{BCE}$ of different sign in the narrow temperature range. 
Dear Editor,

Consider please our manuscript, devoted to some new results obtained during the study of the intensive and extensive barocaloric effects near phase transitions in ferroelectric $\mathrm{NH}_{4} \mathrm{HSO}_{4}$.

We hope that the results will be of interest to readers of Journal of Alloys and Compounds.

Sincerely Yours,

Prof. Igor Flerov

\title{
Conventional and inverse barocaloric effects in ferroelectric $\mathrm{NH}_{4} \mathrm{HSO}_{4}$
}

Mikhail V. Gorev, Ekaterina A. Mikhaleva, Igor N. Flerov, Evgeniy V. Bogdanov

e-mail: gorev@iph.krasn.ru (M.V. Gorev), katerina@iph.krasn.ru (E.A. Mikhaleva), flerov@iph.krasn.ru (I.N. Flerov), evbogdanov@yandex.ru (E.V. Bogdanov)

\begin{abstract}
In this study, the conventional and inverse barocaloric effects (BCE) in ferroelectric

$\mathrm{NH}_{4} \mathrm{HSO}_{4}$ are reported. Maximum extensive and intensive BCE near order-disorder phase transition can be achieved at low pressure $\mathrm{p} \leq 0.1 \mathrm{GPa}$. Large thermal expansion of the crystal lattice plays a very important role in the developing conventional BCE and conversation between $\mathrm{BCE}$ of different sign in the narrow temperature range.
\end{abstract}




\section{Prime Novelty Statement}

Detailed studies of the barocaloric effect at the phase transitions $\mathrm{P} 2{ }_{1} / \mathrm{c}(\mathrm{T} 1) \leftrightarrow \mathrm{Pc}(\mathrm{T} 2) \leftrightarrow \mathrm{P} 1$ in ammonium hydrosulphate were carried out for the first time and showed new peculiar properties of $\mathrm{NH}_{4} \mathrm{HSO}_{4}$.

1) Both ferroelectric transformations are characterized by large baric coefficients of different sign.

2) Hydrostatic pressure strongly decreases the entropy jump at T2 which reaches zero at a pressure of $0.17 \mathrm{GPa}$.

3) Very low pressure is needed to realize the maximum values of the extensive and intensive inverse $\mathrm{BCE}$ at $\mathrm{T} 2$.

4) Large thermal expansion of the crystal lattice leads to two very important points. Firstly, the conventional $\mathrm{BCE}$ at $\mathrm{T} 1$ can be greatly increased to values much higher than the magnitudes corresponding to the entropy of the phase transition. Secondly, in the case of the negative baric coefficient at $\mathrm{T} 2$, a conversion from the inverse to conventional BCE can be realized by low pressure in the narrow temperature range.

5) The results obtained are very important and promising from the point of view of searching materials with large barocaloric effects and designing original cooling cycles. 


\section{Response to Reviewers}

\section{To Reviewer \#1:}

Dear Sir/Madam,

Thank you very much for the efficient comments and appreciation of our paper.

Q1. The final refinement convergence ( $R p$ and $R W p)$ should be given.

OK! We added this information to the description of X-ray structural characterization (page 4):

Instead of

"Fig. 1 shows the results of Rietveld refinement"

we put

"Fig. 1 shows the results of Rietveld refinement $\left(\mathrm{Rwp}=6.04, \mathrm{Rp}=4.23, \mathrm{chi}^{\wedge} 2=\right.$ 2.06)."

Q2. In figure 4, the maximum barocaloric entropy changed slightly, and the peak shifted to low temperature. It seems that the pressure-dependent entropy change neat $T 1$ that arises on heating through the first order phase transition. However, first order phase transition usually exhibits large thermal hysteresis. How about the pressure-dependent entropy change neat T1 on cooling?

Sorry, we think that you mean the transition at T2.

Unfortunately, experimental setups (adiabatic calorimeter and DTA under pressure) do not allow measurements in cooling mode. Therefore, the determination of the BCE was carried out only in the heating mode. The hysteresis of the phase transition temperature $\delta \mathrm{T}_{2} \leq 2.5 \mathrm{~K}$ [Izvestiya AN USSR, 39 (1975) 752-757] is much smaller then temperature interval of BCE at $\mathrm{p}>0.02 \mathrm{GPa}$.

In addition, it's hard to get the maximum adiabatic temperature values, especially under low hydrostatic pressure. Therefore, adiabatic temperature changes between $140 \mathrm{~K}$ and $170 \mathrm{~K}$ under different hydrostatic pressure should be inset in figure $4 b$.

OK! We changed the Figure $4 \mathrm{~b}$ by adding an insert.

Q3. Coexistence of the conventional and inverse barocaloric effects in one material is very intriguing. The negative barocaloric effect may propose novel combinations with conventional (positive) barocaloric effect to further enhance the cooling efficiency. Similar approach has been proposed in electrocaloric materials 
to enhance the coefficient of performance (COP) by combining conventional (positive) and inverse (negative) electrocaloric effects together [Nano Energy (2015) 16, 419]. Is it possible to enhance the COP by combining conventional and inverse barocaloric effects? If it is possible, what is the original cooling cycles?

You are absolutely right. Coexistence of the conventional and inverse barocaloric effects in one material is very intriguing. However, in the case of NH4HSO4, these effects associated with two transitions are too far in temperature (160 and $270 \mathrm{~K})$. In the region near $160 \mathrm{~K}$, the conventional effect due to the change in the entropy of the lattice under pressure is rather small. In our opinion, a more interesting phenomenon can be associated with direct and inverse effects observed at a single transition under the action of uniaxial stresses along different crystallographic axes. We are currently working in this direction. We hope that the results of such studies will be more informative for the analysis of COP. Thank your for the reference on interesting paper. We know it and will use further./

Q4. "This paper demonstrates BCE in NH4HSO4 undergoing two successive ferroelectric phase transitions of displacive and order/disorder type at $T 1$ and at T2, respectively. Both transformations are characterized by large baric coef icients of dif erent sign." This conclusion is not clear. It has been shown that the purely displacive and order-disorder $(O D)$ cases are merely limiting situations, but they are typically thought to describe a majority of the known ferroelectric phase transitions. In the displacive structural phase transformation case, atoms remain associated with their average positions, and phase transition occurs as the positional pattern changes its symmetry. If the corresponding phonon mode frequency decreases to zero near the phase transition temperature, it is a signature of a displacive case (known as soft mode behavior). In the OD case, the structural model involves partially occupied sites, and the transition occurs as the symmetry of the occupational distribution is broken. As a result, the relevant phonon frequency stays temperature independent, while another strongly temperature dependent excitation of the relaxation type occurs. More discussion in the main text or additional experiments should be provided.

Thank you for your comments. Indeed, the mechanism of the structural phase transitions is very often complicated and sometimes can even include the features of both displacive and OD cases. On the other hand, it is known that the entropy as a quantitative characteristic is strongly different for transformations of both types. This situation is observed in the case of NH4HSO4: $\Delta \mathrm{S}_{1}<<\Delta \mathrm{S}_{2}$. However, we 
decided not to discuss such a complex issue, which is not directly related to our research, and make some changes in Conclusions.

Instead of

"This paper demonstrates BCE in NH4HSO4 undergoing two successive ferroelectric phase transitions of displacive and order/disorder type at T1 and at T2, respectively. Both transformations are characterized by large baric coef icients of different sign."

we put

"This paper demonstrates $\mathrm{BCE}$ in $\mathrm{NH}_{4} \mathrm{HSO}_{4}$ undergoing two successive ferroelectric phase transitions characterized by significantly different entropy changes $\left(\Delta \mathrm{S}_{1}=1.2 \mathrm{~J} / \mathrm{mol} \cdot \mathrm{K}, \Delta \mathrm{S}_{2}=7.6 \mathrm{~J} / \mathrm{mol} \cdot \mathrm{K}\right)$ and large baric coefficients of different sign $\left(\mathrm{dT}_{1} / \mathrm{dp}=+90 \mathrm{~K} / \mathrm{GPa}\right.$ and $\left.\mathrm{dT}_{2} / \mathrm{dp}=-123 \mathrm{~K} / \mathrm{GPa}\right) . "$

\section{To Reviewer \#2}

Dear Sir/Madam,

Thank you very much for your comments, questions and appreciation of our paper.

1. What is the mechanism underlying the observation of the inverse barocaloric effects?

Both extensive $\Delta \mathrm{S}_{\mathrm{BCE}}$ and intensive $\Delta \mathrm{T}_{\mathrm{AD}}$ barocaloric parameters strongly depend on the volume thermal expansion $(\partial \mathrm{V} / \partial \mathrm{T})_{\mathrm{p}}$ which very often shows large change near the temperature of the phase transitions. In accordance with Eq. 1, the negative baric coefficient $\mathrm{dT} / \mathrm{dp}$ associated with the negative value $(\partial \mathrm{V} / \partial \mathrm{T})_{\mathrm{p}}$ is the reason of the inverse $\mathrm{BCE}$ at $\mathrm{T}_{2}$ accompanied by increase in entropy and decrease in temperature under pressure increase.

2. Why do the inverse barocaloric effects coexist with the conventional barocaloric effects?

Conventional (near $\mathrm{T}_{1}$ ) and inverse (near $\mathrm{T}_{2}$ ) $\mathrm{BCE}$ are observed at different phase transitions and determined by the sign of the temperature shift of these transitions under pressure: $\mathrm{dT}_{1} / \mathrm{dp}>0, \mathrm{dT}_{2} / \mathrm{dp}<0$ (Fig. 2).

Near $160 \mathrm{~K}$ these effects are associated with different components - with the change in the entropy of the crystal lattice (conventional) and the change in anomalous entropy (inverse, $\mathrm{dT} 2 / \mathrm{dp}<0$ ). 
3. The maximum barocaloric effects were observed near the order-disorder phase transition temperature, where the heat capacity $C p$ should also reach the maximum value. It would be better if the authors could measure heat capacity at various temperatures.

The detailed heat capacity studies at $\mathrm{p}=0$ was performed by us earlier in wide temperature range [19], where both the lattice and anomalous components of heat capacity and entropy were determined. Using these data, the values of barocaloric effects were determined at the first stage (Fig. 3-4 (a, b)). At the second stage, we took into account the dependence of the entropy of the crystal lattice on pressure using the data on thermal expansion (Fig. 4 (c, d)). And finally, using the DTA data under pressure, we were able to take into account the pressure dependence of the entropy jump at the first-order phase transition at $\mathrm{T}_{2}$ (Fig. 5).

4. The authors might discuss the potential application of the coexistence of the inverse barocaloric effect and the conventional barocaloric effect. An idea has been proposed to improve the cooling efficiency in ferroelectrics via combining inverse (negative) and conventional (positive) electrocaloric effects [Nano Energy 16, 419-427 (2015)].

Thank your for the reference on interesting paper. We know it and will use in our further researches.

You are absolutely right. Coexistence of the conventional and inverse barocaloric effects in one material is very intriguing. However, in the case of NH4HSO4, these effects associated with two transitions are too far in temperature (160 and $270 \mathrm{~K})$. In the region near $160 \mathrm{~K}$, the conventional effect due to the change in the entropy of the lattice under pressure is rather small. In our opinion, a more interesting phenomenon can be associated with direct and inverse effects observed at a single transition under the action of uniaxial stresses along different crystallographic axes. We are currently working in this direction. We hope that the results of such studies will be more informative for the analysis of COP.

Thank you again.

Best regards, Igor Flerov. 


\section{Response to Reviewers}

\section{To Reviewer \#1:}

Dear Sir/Madam

Thank you very much for your positive final decision on our paper.

Best regards, Igor Flerov.

\section{To Reviewer \#3:}

Dear Sir/Madam

Thank you very much for your positive final decision on our paper.

Best regards, Igor Flerov.

\section{To Reviewer \#4:}

Dear Sir/Madam,

Tank you for your interesting question on lattice constants change with pressure and temperature.

Indeed, such information would be useful in our analysis of BCE.

Unfortunately, firstly, such data are not available in the literature and, secondly, we were not able to perform such studies due to the lack of appropriate equipment.

On the other hand, we took into account the data on the behaviour of lattice constants of related (NH4)2SO4 [15] where no noticeable effect of pressure on the thermal expansion of the lattice was found.

Please see page 8 in the manuscript:

"Taken into account the data of the thermal expansion study of the related (NH4)2SO4 [15], it was suggested that the values of $\beta_{L A T}$ and $V_{m}$ of NH4HSO4 are also weakly depend on the pressure."

In connection with the above, we did not make any corrections and/or additions in the text of the manuscript. 
Thank you very much for your positive final decision on our paper.

Best regards,

Igor Flerov. 


\title{
Conventional and inverse barocaloric effects in ferroelectric $\mathrm{NH}_{4} \mathrm{HSO}_{4}$
}

\author{
Mikhail V. Gorev ${ }^{\mathrm{a}, \mathrm{b}}$, Ekaterina A. Mikhaleva, ${ }^{\mathrm{a}, \mathrm{b}}$, Igor N. Flerov ${ }^{\mathrm{a}, \mathrm{b}, *}$, Evgeniy \\ V. Bogdanov ${ }^{a, c}$ \\ ${ }^{a}$ Kirensky Institute of Physics, Federal Research Center KSC SB RAS, Krasnoyarsk, Russia \\ ${ }^{b}$ Institute of Engineering Physics and Radioelectronics, Siberian Federal University, \\ Krasnoyarsk, Russia \\ ${ }^{c}$ Institute of Engineering Systems and Energy, Krasnoyarsk State Agrarian University, \\ 660049 Krasnoyarsk, Russia
}

\begin{abstract}
In this study, the conventional and inverse barocaloric effects (BCE) in ferroelectric $\mathrm{NH}_{4} \mathrm{HSO}_{4}$ are reported. Maximum extensive and intensive $\mathrm{BCE}$ near order-disorder phase transition can be achieved at low pressure $p \leq 0.1 \mathrm{GPa}$. Large thermal expansion of the crystal lattice plays a very important role in the developing conventional BCE and conversation between BCE of different sign in the narrow temperature range.
\end{abstract}

Keywords: Polymorphic phase transformation, Phase diagram, Order-disorder phenomena, Entropy, Barocaloric effect

PACS: 62.50.-p, 65.40.-b, 81.30.-t

\section{Introduction}

In recent years, much attention is paid to caloric effects (CE) in solids, particularly in ferroics, associated with the reversible change in the temperature, $\Delta T_{A D}$, or entropy, $\Delta S_{C E}$, under variation of the external field in adiabatic and isothermal conditions, respectively [1, 2, 3, 4]. One of the main reasons for this interest is related to the possibility to use the materials showing large CE's

\footnotetext{
* Corresponding author

Email addresses: gorev@iph.krasn.ru (Mikhail V. Gorev), katerina@iph.krasn.ru (Ekaterina A. Mikhaleva), flerov@iph.krasn.ru (Igor N. Flerov), evbogdanov@iph.krasn.ru (Evgeniy V. Bogdanov)
}

Preprint submitted to Journal of Alloys and Compounds

July 2, 2019 
as solid state refrigerants in alternative cooling cycles [5, 6, 7, 8, Among the CEs of different physical nature, the barocaloric effect (BCE) is distinguished by a serious advantage associated with its universality. Indeed, both extensive $\Delta S_{B C E}$ and intensive $\Delta T_{A D}$ barocaloric parameters strongly depend on the volume thermal expansion $(\partial V / \partial T)_{p}$ which very often shows large change near the temperature of any phase transitions: ferroelectric, ferroelastic, ferromagnetic

$$
\Delta S_{B C E}=-\int_{0}^{p}\left(\frac{\partial V}{\partial T}\right)_{p} d p, \Delta T_{A D}=-\frac{T}{C_{p}} \Delta S_{B C E}
$$

where $C_{p}$ is the heat capacity.

The most intensively, BCE was studied in materials undergoing ferroelastic [9, 10] and ferromagnetic [11, 12, 13, 14, phase transitions. As to the ferro5 electrics, their barocaloric efficiency was investigated only sporadically [11, 15, [16, 17]. It is known that the values and behavior of the BCE depend on the behavior and change in the entropy $\Delta S$ of the phase transition as well as on the sensitivity of the phase transition temperature to hydrostatic pressure [9, 10]. Thus, ferroics undergoing order-disorder transformations accompanied by large change in the volume and as result in baric coefficient, $d T_{0} / d p=\delta V / \delta S$, are the most promising barocaloric materials. Important requirements for caloric materials are also their low cost and ecological tolerance.

It has recently been shown that ferrielectric $\left(\mathrm{NH}_{4}\right)_{2} \mathrm{SO}_{4}$ meets all of the above requirements [15. Due to significant values of $\Delta S=17 \mathrm{~J} / \mathrm{mol} \cdot \mathrm{K} \approx R \ln 8$ and $d T_{0} / d p=-45 \mathrm{~K} / \mathrm{GPa}$, rather large extensive and intensive BCE were observed in the region of the phase transition Pnam - Pna $2_{1}$ under low pressure. In accordance with Eq. 1, the negative baric coefficient associated with the negative value $(\partial V / \partial T)_{p}$ is the reason of the inverse $\mathrm{BCE}_{i n v}$ in $\left(\mathrm{NH}_{4}\right)_{2} \mathrm{SO}_{4}$ accompanied by increase/decrease in entropy/temperature under pressure increase. It was also found that large coefficient of the volume thermal expansion of the crystal lattice, $\beta_{L A T}$, can play an important role in formation of real $\mathrm{BCE}$ in material. Indeed, in the case of ammonium sulphate, large positive value $\beta_{L A T}=1.4 \times 10^{-4} \mathrm{~K}^{-1}$ leads to decrease in the inverse $\mathrm{BCE}_{\text {inv }}$ under pressure due to the appearance of the conventional contribution BCE $\left(\triangle S_{B C E}<0\right.$, 
$\left.\Delta T_{A D}>0\right)$ [15]. The conversion from $\mathrm{BCE}_{i n v}$ to $\mathrm{BCE}_{\text {conv }}$ was observed in a narrow temperature range. When pressure increases, the ratio between these values changes and at $p=0.25 \mathrm{GPa}$ is about $\mathrm{BCE}_{\text {conv }} / \mathrm{BCE}_{i n v}=0.15$. Thus, to get correct information on $\mathrm{BCE}$ in materials with large thermal expansion coefficient, it is necessary to take into account the effect of pressure on the lattice entropy. It is obvious that the magnitude of baric coefficient strongly effects on the maximum value of the intensive $\mathrm{BCE}$ [18. In this respect, it is interesting to analyze both BCE in material with anomalously large negative or positive $d T / d p$. From this point of view, another ferroelectric crystal, ammonium hydrogen sulphate, is very good example.

Indeed, $\mathrm{NH}_{4} \mathrm{HSO}_{4}$ undergoes two successive phase transitions $P 2_{1} / c \leftrightarrow$ $P c \leftrightarrow P 1$, of the strong second and first order at $T_{1}=271 \mathrm{~K}$ and $T_{2}=159 \mathrm{~K}$, respectively. One more difference is that anomaly of volumetric thermal expansion coefficient is positive at $T_{1}$ and negative at $T_{2}$ [19] which leads to $\mathrm{BCE}_{\text {conv }}$ and $\mathrm{BCE}_{i n v}$. Thus, the contribution from the thermal expansion of the crystal

40 lattice to both BCE will be also different. Despite the large difference in the entropy of the phase transitions $\left(\Delta S_{1}=1.2 \mathrm{~J} / \mathrm{mol} \cdot \mathrm{K}, \Delta S_{2}=7.6 \mathrm{~J} / \mathrm{mol} \cdot \mathrm{K}\right)$, one can suppose that $\mathrm{BCE}_{\text {conv }}$ at $T_{1}$ could be strongly increased due to rather large value of $\beta_{L A T}=2 \times 10^{-4} \mathrm{~K}^{-1}$ far from the phase transition points. In addition, like $\left(\mathrm{NH}_{4}\right)_{2} \mathrm{SO}_{4}$, ammonium hydrogen sulphate is also easy to prepare, cheap 45 and environmentally friendly.

In the present paper, we performed an analysis of extensive and intensive barocaloric efficiency of $\mathrm{NH}_{4} \mathrm{HSO}_{4}$ near both phase transformations based on methods developed by us earlier [18. For this aim, the dependencies of $\Delta T_{A D}(T, p)$ and $\Delta S_{B C E}(T, p)$ were determined using data on total and anomalous heat capacity [19], the $T-p$ phase diagram and the dependencies of entropy of the phase transitions on temperature and pressure. 


\section{Experimental details}

Powder samples of ammonium hydrogen sulphate were obtained by slow evaporation at $45^{\circ} \mathrm{C}$ from an aqueous solution containing equimolar quantities of high purity raw materials $\left(\mathrm{NH}_{4}\right)_{2} \mathrm{SO}_{4}$ and $\mathrm{H}_{2} \mathrm{SO}_{4}$.

The quality of samples used for the experiments was checked at room temperature using XRD, which revealed a monoclinic symmetry consistent with the space group $P 2_{1} / c(Z=8)$ suggested in Refs. [20, 21]. No additional phases were observed in the samples. Fig. 1 shows the results of Rietveld refinement $\left(R_{w p}=6.04, R_{p}=4.23, \chi^{2}=2.06\right)$. The unit cell parameters $a=24.770(6) \AA$, $b=4.611(1) \AA, c=14.871(4) \AA, \beta=89.70(1) \operatorname{grad}$ are consistent with the values determined in Ref. [21].

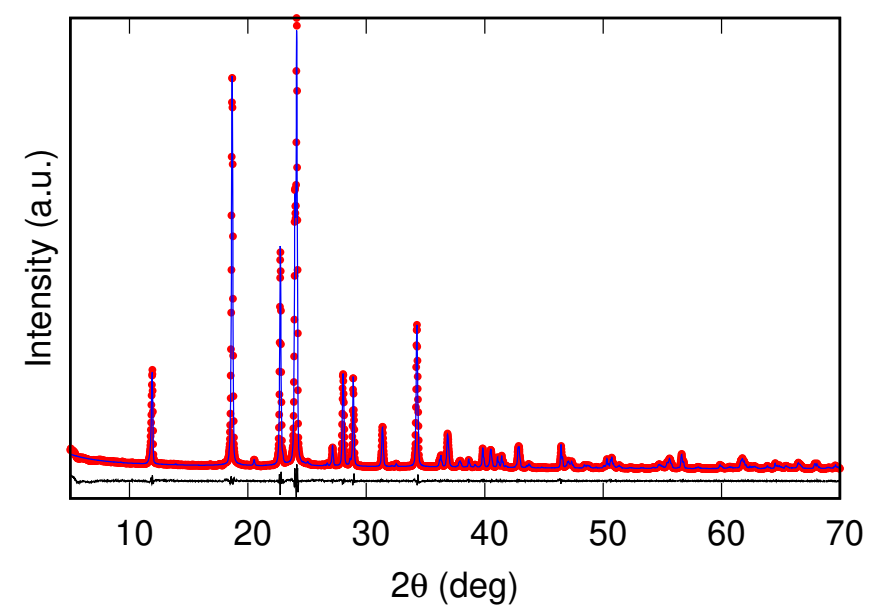

Figure 1: Difference Rietveld plot for $\mathrm{NH}_{4} \mathrm{HSO}_{4}$ at room temperature.

Quasi-ceramic samples of $\mathrm{NH}_{4} \mathrm{HSO}_{4}$ in the form of disks, approximately $1.0 \mathrm{~mm}$ thick and approximately $6 \mathrm{~mm}$ in diameter, were used for investigations. Because of the presence of ammonium ion in crystal, the heat treatments of ceramics were not performed. For dielectric measurements, electrodes on pellets were formed by conducting glue covered the opposite sides of the sample.

The effect of hydrostatic pressure on temperature and entropy of the phase transitions was studied using a piston-cylinder type vessel associated with a 
pressure multiplier. Pressure of up to $0.25 \mathrm{GPa}$ was generated using a mixture of silicon oil and pentane exhibiting optimal electrical and heat conductivity, solidification point and viscosity as the pressure-transmitting medium. Pressure and temperature were measured using a manganin resistive sensor and a copperconstantan thermocouple, with accuracies of about $\pm 10^{-3} \mathrm{GPa}$ and $\pm 0.3 \mathrm{~K}$ 75 respectively.

The dependencies $T_{1}(p)$ and $T_{2}(p)$ were revealed, firstly, in experiments with differential thermal analysis (DTA) and, secondly, by measurements of the permittivity $\varepsilon$. In the former case, to detect anomalies of DTA-signal associated with the heat capacity anomalies, high-sensitive differential copper-germanium thermocouple was used. In the latter case, experiments were performed using an E7-20 immittance meter. To ensure the reliability of the results, the measurements were performed for both increasing and decreasing pressure cycles.

\section{Results and discussion}

At ambient pressure, the anomalies of DTA signal and $\varepsilon$ were detected at about $T_{1}=271.5 \pm 1.0 \mathrm{~K}$ and $T_{2}=160 \pm 2 \mathrm{~K}$ (Fig.2(a) and (b)), which agree well with values observed during measurements of the heat capacity [19]. Fig. 2(c) shows that an increase in pressure leads to linear increase and decrease in $T_{1}$ and $T_{2}$, respectively: $d T_{1} / d p=+90 \pm 15 \mathrm{~K} / \mathrm{GPa}$ and $d T_{2} / d p=-123 \pm 15 \mathrm{~K} / \mathrm{GPa}$ which are significantly higher than that for ammonium sulfate [15].

Due to the limited sensitivity of the DTA method, the area under the DTA peak at $T_{2}$ represents a change in the enthalpy $\delta H_{2}$ (entropy $\left.\delta S_{2}=\delta H_{2} / T_{2}\right)$ jump at the first order phase transition $P c \leftrightarrow P 1$ in $\mathrm{NH}_{4} \mathrm{HSO}_{4}$. An increase in pressure is accompanied by a linear decrease in the value of $\delta S_{2}$ which reaches zero at $p \approx 0.17 \mathrm{GPa}$ (Fig. 2(d)) that can be considered as corresponding to the pressure of the tricritical point. On the other hand, it is unlikely that such a low pressure may affect the degree of disordering of structural elements in phases $P 2_{1} / c$ and $P c$, and as a result the total entropy change at the $P c \leftrightarrow P 1$ transformation, $\Delta S_{2}(p)=\delta S_{2}(p)+\Delta S_{2}^{*}(T, p)$, remains constant. Here $\Delta S_{2}^{*}(T, p)$ is 

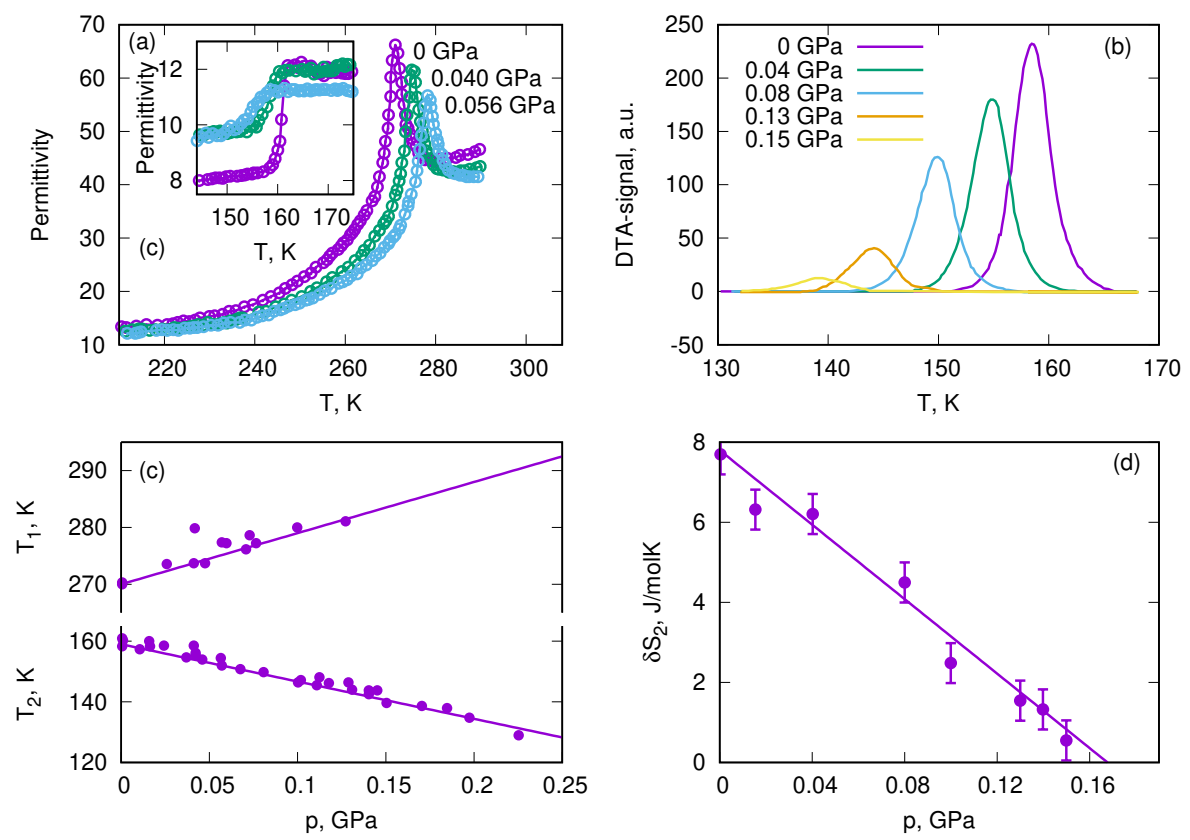

Figure 2: (a) Temperature dependencies of permittivity for $\mathrm{NH}_{4} \mathrm{HSO}_{4}$ around $T_{1}$ and $T_{2}$ and (b) anomalous component of the DTA signal near $T_{2}$ at different hydrostatic pressure. (c) Temperature - pressure phase diagram combining the results on the DTA signal and permittivity study. (d) Entropy jump $\delta S_{2}$ for the first-order transition in $\mathrm{NH}_{4} \mathrm{HSO}_{4}$ at different hydrostatic pressure.

the temperature- and pressure-dependent contribution.

The analysis of extensive and intensive $\mathrm{BCE}$ in $\mathrm{NH}_{4} \mathrm{HSO}_{4}$ was performed in three steps.

At the first stage, the possible influence of pressure on the entropy of the crystal lattice $\Delta S_{L A T}$ was not taken into account. Fig. 3 demonstrates the temperature behavior of the lattice $S_{L A T}(T)-S_{L A T}(100 \mathrm{~K})=\int_{100}^{T}\left(C_{L A T} / T\right) d T$ and total $S=\int_{100}^{T}\left(C_{p} / T\right) d T$ entropies in the vicinities of $T_{1}$ and $T_{2}$. Temperature dependencies $S(T)$ under pressure were determined by summation of the lattice entropy $S_{L A T}$ and the anomalous contributions $\Delta S_{1}$ and $\Delta S_{2}$ shifted along the temperature scale in accordance with the sign of baric coefficients $d T_{1} / d p$ and $d T_{2} / d p$. The values and behavior of extensive BCE, $\triangle S_{B C E}$, at different pressure were determined from temperature dependencies of the total 

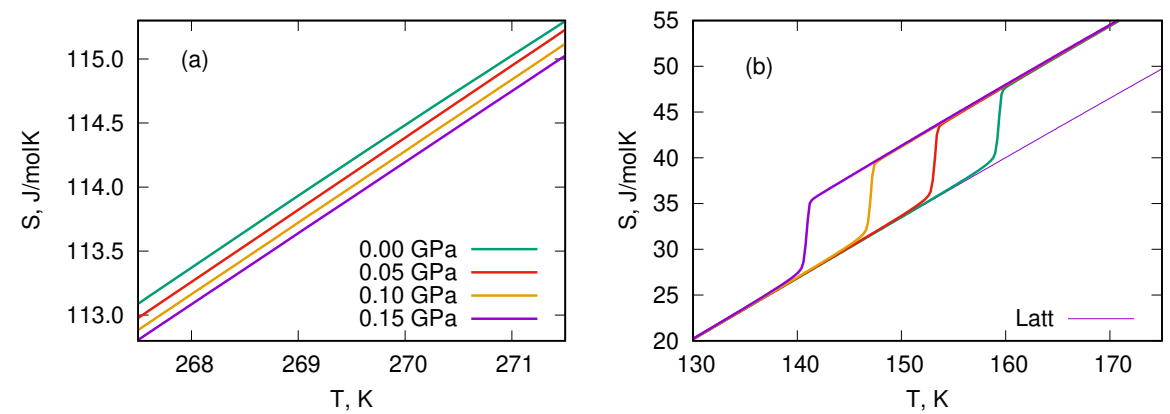

Figure 3: Temperature dependencies of total entropy of $\mathrm{NH}_{4} \mathrm{HSO}_{4}$ at different hydrostatic pressure near (a) $T_{1}$ and (b) $T_{2}$.

entropy as a difference $\Delta S_{B C E}=S(T, p)-S(T, p=0)$ at constant temperature (Fig. $4(\mathrm{a})$ ). The temperature dependencies of the intensive BCE were revealed analyzing plots of $S(T, p)=S_{L A T}(T, p=0)+\Delta S(T, p)$ at constant entropy $S(T, p)=S\left(T+\Delta T_{A D}, p=0\right)$ (Fig. $\left.4(\mathrm{~b})\right)$. Large difference in BCE at $T_{1}$ and $T_{2}$ at the same pressure is the result of the different values of $\Delta S_{1}$ and $\Delta S_{2}$.

It is known 22 that if we neglect the contribution of the thermal expansion of the crystal lattice, the maximum values of both $\mathrm{BCE}$ are limited by the value of the entropy of the phase transition: $\left(\triangle S_{B C E}^{\max }\right)_{T 1}=\Delta S_{1}=-10 \mathrm{~J} / \mathrm{kg} \cdot \mathrm{K}$, $\left(\Delta T_{A D}^{\max }\right)_{T 1}=2 \mathrm{~K} ;\left(\Delta S_{B C E}^{\max }\right)_{T 2}=\Delta S_{2}=68 \mathrm{~J} / \mathrm{kg} \cdot \mathrm{K},\left(\Delta T_{A D}^{\max }\right)_{T 2}=12 \mathrm{~K}$. However, undoubtedly important is the ability to realize in the material maximum values of both BCE at low pressure.

Estimates made using the following relation $p_{\min }=T \Delta S /\left(C_{L A T} d T / d p\right)$, valid for phase transitions of the first order $[9]$, show that the value $\left(\triangle S_{B C E}^{\max }\right)_{T 2}$ can be implemented in $\mathrm{NH}_{4} \mathrm{HSO}_{4}$ by rather insignificant pressure $0.1 \mathrm{GPa}$. In fact, the rather large value $\left(\Delta S_{B C E}\right)_{T 2}=0.95\left(\Delta S_{B C E}^{\max }\right)_{T 2}$ can be achieved even at much lower pressure, $p \approx 0.02 \mathrm{GPa}$ (Fig. 4(a)). However, intensive $\mathrm{BCE}$, which also depends on the $d S_{L A T} / d T$ derivative, is characterized by lower increase rate under pressure and reaches $\left(\Delta T_{A D}^{\max }\right)_{T 2}$ at about $0.13 \mathrm{GPa}$ (Fig. $4(\mathrm{~b})$ ). As to the second order phase transition, at pressure 0.1 GPa exten130 sive and intensive effects reach only about $18 \%$ of $\left(\Delta S_{B C E}^{\max }\right)_{T 1}$ and $\left(\Delta T_{A D}^{\max }\right)_{T 1}$.

At the second stage, effect of the lattice entropy change under pressure 

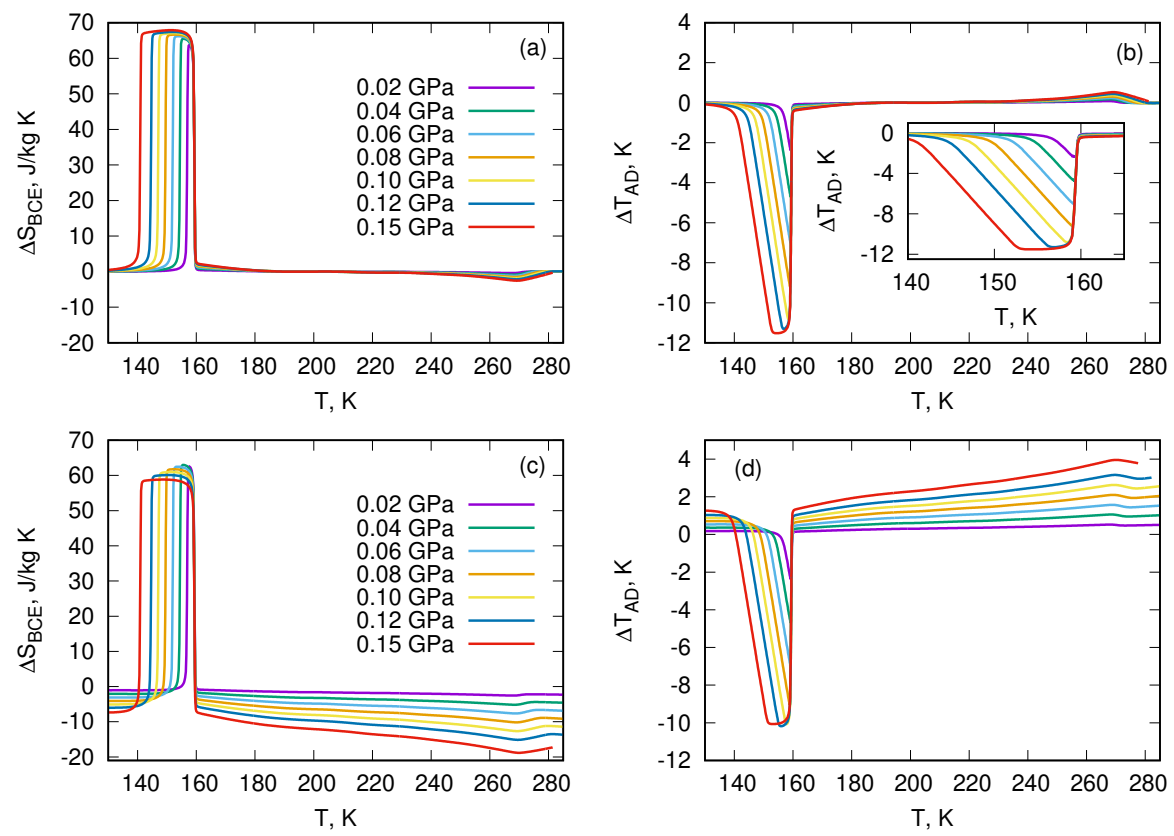

Figure 4: (a) Barocaloric entropy and (b) adiabatic temperature changes at different hydrostatic pressure in a wide temperature range determined without taking into account the effect of thermal expansion of the crystal lattice. Effect of thermal expansion of the crystal lattice on (c) $\triangle S_{B C E}$ and (d) $\Delta T_{A D}$

on $\mathrm{BCE}$ in $\mathrm{NH}_{4} \mathrm{HSO}_{4}$ was studied. This contribution can be evaluated using Maxwell relation $\left(\partial S_{L A T} / \partial p\right)_{T}=-(\partial V / \partial T)_{p}$

$$
\Delta S_{L A T}(T, p)=-\int_{0}^{p}(\partial V / \partial T)_{p} d p \approx-V_{m} \beta_{L A T}(T) p .
$$

Here $\left.V_{m}=6.17 \times 10^{-5} \mathrm{~m}^{3} / \mathrm{mol}\right)$ is the molar volume.

Taken into account the data of the thermal expansion study of the related $\left(\mathrm{NH}_{4}\right)_{2} \mathrm{SO}_{4}$ [15, it was suggested that the values of $\beta_{L A T}$ and $V_{m}$ of $\mathrm{NH}_{4} \mathrm{HSO}_{4}$ are also weakly depend on the pressure. Lattice contribution was determined from the results of dilatometric study of $\mathrm{NH}_{4} \mathrm{HSO}_{4}$ [19].

Fig. 4(c) and (d) demonstrate that due to the same sign of both derivatives, $(\partial V / \partial T)_{T 1}$ and $\left(\partial V_{L A T} / \partial T\right)$, there is a strong increase in $\mathrm{BCE}_{c o n v}$. At $p=0.15 \mathrm{GPa}$, the values $\left(\Delta S_{B C E}\right)_{T 1}=-18.8 \pm 1.5 \mathrm{~J} / \mathrm{kg} \cdot \mathrm{K}$ and $\left(\Delta T_{A D}\right)_{T 1}=$ $4.0 \pm 0.2 \mathrm{~K}$ are about one and a half times higher than even the maximum 
Thus, contributions of $\left(\Delta S_{B C E}^{L A T}\right)_{T 1}$ and $\left(\Delta T_{A D}^{L A T}\right)_{T 1}$ to the full BCE at $T_{1}$ are predominant.

On the other hand, at the same pressure, $\mathrm{BCE}_{i n v}$ associated with the phase transition $P c-P 1\left((\partial V / \partial T)_{T 2}<0\right)$, is reduced to the magnitudes $\left(\Delta S_{B C E}\right)_{T 2}=$ $59 \pm 5 \mathrm{~J} / \mathrm{kg} \cdot \mathrm{K},\left(\Delta T_{A D}\right)_{T 2}=-10.0 \pm 0.8 \mathrm{~K}$ by $\mathrm{BCE}_{\text {conv }}^{L A T}$ arising in accordance with Eq. 2. $\left(\partial V_{L A T} / \partial T\right)>0:\left(\Delta S_{B C E}^{L A T}\right)_{T 2}=-9.0 \pm 0.7 \mathrm{~J} / \mathrm{kg} \cdot \mathrm{K},\left(\Delta T_{A D}^{L A T}\right)_{T 2}=$ $1.5 \pm 0.1 \mathrm{~K}$.

At last, at the third stage, we determine the behavior of extensive and intensive $\mathrm{BCE}$ at $T_{2}$ taken into account the peculiarities of experiments with DTA under pressure. As it was discussed above, increase in pressure strongly decreases the entropy jump $\delta S_{2}$ at the first order phase transition $P c-P 1$ (Fig. 2(b) and (d)), while the total entropy change $\Delta S_{2}$ remains constant. Analyzing the dependencies $S(T, p)$ using both the DTA data under pressure and the effect of the lattice expansion, barocaloric parameters at $T_{2}$ are determined and One can see that in this case the magnitudes of $\left(\Delta S_{B C E}^{\max }\right)_{T 2}$ and $\left(\Delta T_{A D}^{\max }\right)_{T 2}$ are realized at almost the same low pressure which was determined in the second stage (Fig. 4(c) and (d)). The main difference was observed in the form of peaks $\left(\Delta S_{B C E}\right)_{T 2}(T)$ and $\left(\Delta T_{A D}\right)_{T 2}(T)$ which is due to peculiarities of the processes of measuring the heat capacity by methods of adiabatic calorimeter and DTA.

Returning to the results of the BCE study at $T_{1}$, one can confidently argue that it would be interesting and useful to investigate the influence of the thermal expansion of the crystal lattice on conventional BCE in ferroelectrics characterized by large $\beta_{L A T}$ and undergoing order-disorder transformation with

\section{Conclusion}

This paper demonstrates $\mathrm{BCE}$ in $\mathrm{NH}_{4} \mathrm{HSO}_{4}$ undergoing two successive ferroelectric phase transitions characterized by significantly different entropy changes 

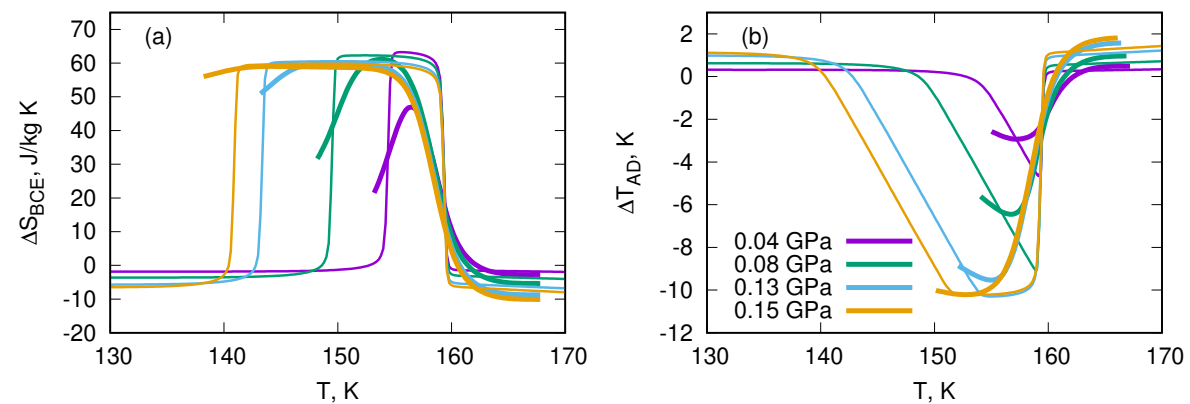

Figure 5: (a) Barocaloric entropy and (b) adiabatic temperature changes at different hydrostatic pressure determined using DTA data on a jump of the entropy (thick lines) and data presented in Fig, 4(c) and (d) (thin lines).

$\left(\Delta S_{1}=1.2 \mathrm{~J} / \mathrm{mol} \cdot \mathrm{K}, \Delta S_{2}=7.6 \mathrm{~J} / \mathrm{mol} \cdot \mathrm{K}\right)$ and large baric coefficients of different sign $\left(d T_{1} / d p=+90 \mathrm{~K} / \mathrm{GPa}\right.$ and $\left.d T_{2} / d p=-123 \mathrm{~K} / \mathrm{GPa}\right)$. Hydrostatic pressure strongly decreases the entropy jump at $T_{2}$ which reaches zero at a pressure of $0.17 \mathrm{GPa}$. Very low pressure is needed to realize the maximum values of the extensive and intensive inverse BCE. Large thermal expansion of the crystal lattice leads to two very important points. Firstly, the conventional BCE can be greatly increased to values much higher than the magnitudes corresponding to the entropy of the phase transition. Secondly, in the case of the negative baric coefficient, a conversion from the inverse to conventional BCE can be realized by low pressure in the narrow temperature range.

\section{References}

[1] K. A. GschneidnerJr, V. K. Pecharsky, A. O. Tsokol, Recent developments in magnetocaloric materials, Rep. Prog. Phys. 68 (6) (2005) 1479-1539. doi $: 10.1088 / 0034-4885 / 68 / 6 / r 04$.

[2] M. Valant, Electrocaloric materials for future solid-state refrigeration technologies, Prog. Mater. Sci. 57 (6) (2012) 980 - 1009. doi:10.1016/j. pmatsci.2012.02.001. 
[3] X. Moya, S. Kar-Narayan, N. D. Mathur, Caloric materials near ferroic phase transitions, Nat. Mater. 13 (2014) 439-450. doi:10.1038/nmat3951

[4] H. Khassaf, T. Patel, R. J. Hebert, S. P. Alpay, Flexocaloric response of epitaxial ferroelectric films, J. Appl. Phys. 123 (2) (2018) 024102. doi: $10.1063 / 1.5009121$

[5] U. Tomc, J. Tušek, A. Kitanovski, A. Poredoš, A new magnetocaloric refrigeration principle with solid-state thermoelectric thermal diodes, Appl. Thermal Engineering 58 (1) (2013) 1-10. doi:10.1016/j. applthermaleng.2013.03.063.

[6] U. Plaznik, M. Vrabelj, Z. Kutnjak, B. Malič, A. Poredoš, A. Kitanovski, Electrocaloric cooling: The importance of electric-energy recovery and heat regeneration, EPL (Europhysics Letters) 111 (5) (2015) 57009. doi:10. 1209/0295-5075/111/57009

[7] A. Kitanovski, U. Plaznik, U. Tomc, A. Poredo, Present and future caloric refrigeration and heat-pump technologies, Int. J. Refrigeration 57 (2015) 288 - 298. doi:10.1016/j.ijrefrig.2015.06.008.

[8] N. Michaelis, F. Welsch, S.-M. Kirsch, M. Schmidt, S. Seelecke, A. Schütze, Experimental parameter identification for elastocaloric air cooling, Int. J.

(1) Refrigeration 100 (2019) 167 - 174. doi:10.1016/j.ijrefrig.2019.01. 006.

[9] M. Gorev, E. Bogdanov, I. Flerov, T-p phase diagrams and the barocaloric effect in materials with successive phase transitions, J. Phys. D: Appl. Phys. 50 (38) (2017) 384002. doi:10.1088/1361-6463/aa8025

[10] M. Gorev, E. Bogdanov, I. Flerov, Conventional and inverse barocaloric effects around triple points in ferroelastics $\left(\mathrm{NH}_{4}\right)_{3} \mathrm{NbOF}_{6}$ and $\left(\mathrm{NH}_{4}\right)_{3} \mathrm{TiOF}_{5}$,

口 Scripta Materialia 139 (2017) 53-57. doi:10.1016/j.scriptamat.2017. 06.022 
[11] E. Mikhaleva, I. Flerov, A. Kartashev, M. Gorev, A. Cherepakhin, K. Sablina, N. Mikhashenok, N. Volkov, A. Shabanov, Caloric effects

[12] K. Alex Müller, F. Fauth, S. Fischer, M. Koch, A. Furrer, P. Lacorre, Cooling by adiabatic pressure application in $\mathrm{Pr}_{1}-\mathrm{xLaxNiO}$, Appl. Phys.

[13] T. Strässle, A. Furrer, Z. Hossain, C. Geibel, Magnetic cooling by the application of external pressure in rare-earth compounds, Phys. Rev. B 67 (2003) 054407. doi:10.1103/PhysRevB.67.054407.

[14] N. A. de Oliveira, Barocaloric effect and the pressure induced solid state re-

[16] H. Khassaf, T. Patel, S. P. Alpay, Combined intrinsic elastocaloric and electrocaloric properties of ferroelectrics, J. Appl. Phys. 121 (14) (2017) 144102. doi:10.1063/1.4980098.

[17] Y. Liu, J. Wei, P.-E. Janolin, I. C. Infante, X. Lou, B. Dkhil, Giant roomtemperature barocaloric effect and pressure-mediated electrocaloric effect 235 in $\mathrm{BaTiO}_{3}$ single crystal, Appl. Phys. Letters 104 (16) (2014) 162904. doi: $10.1063 / 1.4873162$

[18] M. V. Gorev, I. N. Flerov, E. V. Bogdanov, V. N. Voronov, N. M. Laptash, Barocaloric effect near the structural phase transition in the 口 $\mathrm{Rb}_{2} \mathrm{KTiOF}_{5}$ oxyfluoride, Phys. Solid State 52 (2) (2010) 377-383. doi: $10.1134 / \mathrm{S} 1063783410020253$ 
[19] E. Mikhaleva, I. Flerov, A. Kartashev, M. Gorev, E. Bogdanov, V. Bondarev, Thermal, dielectric and barocaloric properties of $\mathrm{NH}_{4} \mathrm{HSO}_{4}$ crystallized from an aqueous solution and the melt, Solid State Sciences 67 (2017) 1-7. doi:10.1016/j.solidstatesciences.2017.03.004.

[20] R. Pepinsky, K. Vedam, S. Hoshino, Y. Okaya, Ammonium hydrogen sulfate: A new ferroelectric with low coercive field, Phys. Rev. 111 (1958) 1508-1510. doi:10.1103/PhysRev.111.1508.

[21] D. Swain, V. S. Bhadram, P. Chowdhury, C. Narayana, Raman and x-ray investigations of ferroelectric phase transition in $\mathrm{NH}_{4} \mathrm{HSO}_{4}$, J. Phys. Chem. A 116 (2012) 223-230. doi:10.1021/jp2075868

[22] R. Pirc, Z. Kutnjak, R. Blinc, Q. M. Zhang, Upper bounds on the electrocaloric effect in polar solids, Appl. Phys. Letters 98 (2) (2011) 021909. doi:10.1063/1.3543628, 


\title{
Conventional and inverse barocaloric effects in ferroelectric $\mathrm{NH}_{4} \mathrm{HSO}_{4}$
}

\author{
Mikhail V. Gorev ${ }^{\mathrm{a}, \mathrm{b}}$, Ekaterina A. Mikhaleva, ${ }^{\mathrm{a}, \mathrm{b}}$, Igor N. Flerov ${ }^{\mathrm{a}, \mathrm{b}, *}$, Evgeniy \\ V. Bogdanov ${ }^{a, c}$ \\ ${ }^{a}$ Kirensky Institute of Physics, Federal Research Center KSC SB RAS, Krasnoyarsk, Russia \\ ${ }^{b}$ Institute of Engineering Physics and Radioelectronics, Siberian Federal University, \\ Krasnoyarsk, Russia \\ ${ }^{c}$ Institute of Engineering Systems and Energy, Krasnoyarsk State Agrarian University, \\ 660049 Krasnoyarsk, Russia
}

\begin{abstract}
In this study, the conventional and inverse barocaloric effects (BCE) in ferroelectric $\mathrm{NH}_{4} \mathrm{HSO}_{4}$ are reported. Maximum extensive and intensive $\mathrm{BCE}$ near order-disorder phase transition can be achieved at low pressure $p \leq 0.1 \mathrm{GPa}$. Large thermal expansion of the crystal lattice plays a very important role in the developing conventional BCE and conversation between BCE of different sign in the narrow temperature range.
\end{abstract}

Keywords: Polymorphic phase transformation, Phase diagram, Order-disorder phenomena, Entropy, Barocaloric effect

PACS: 62.50.-p, 65.40.-b, 81.30.-t

\section{Introduction}

In recent years, much attention is paid to caloric effects (CE) in solids, particularly in ferroics, associated with the reversible change in the temperature, $\Delta T_{A D}$, or entropy, $\Delta S_{C E}$, under variation of the external field in adiabatic and isothermal conditions, respectively [1, 2, 3, 4]. One of the main reasons for this interest is related to the possibility to use the materials showing large CE's

\footnotetext{
* Corresponding author

Email addresses: gorev@iph.krasn.ru (Mikhail V. Gorev), katerina@iph.krasn.ru (Ekaterina A. Mikhaleva), flerov@iph.krasn.ru (Igor N. Flerov), evbogdanov@iph.krasn.ru (Evgeniy V. Bogdanov)
}

Preprint submitted to Journal of Alloys and Compounds 
as solid state refrigerants in alternative cooling cycles [5, 6, 7, 8, Among the CEs of different physical nature, the barocaloric effect (BCE) is distinguished by a serious advantage associated with its universality. Indeed, both extensive $\Delta S_{B C E}$ and intensive $\Delta T_{A D}$ barocaloric parameters strongly depend on the volume thermal expansion $(\partial V / \partial T)_{p}$ which very often shows large change near the temperature of any phase transitions: ferroelectric, ferroelastic, ferromagnetic

$$
\Delta S_{B C E}=-\int_{0}^{p}\left(\frac{\partial V}{\partial T}\right)_{p} d p, \Delta T_{A D}=-\frac{T}{C_{p}} \Delta S_{B C E}
$$

where $C_{p}$ is the heat capacity.

The most intensively, BCE was studied in materials undergoing ferroelastic [9, 10] and ferromagnetic [11, 12, 13, 14, phase transitions. As to the ferro5 electrics, their barocaloric efficiency was investigated only sporadically [11, 15, [16, 17]. It is known that the values and behavior of the BCE depend on the behavior and change in the entropy $\Delta S$ of the phase transition as well as on the sensitivity of the phase transition temperature to hydrostatic pressure [9, 10]. Thus, ferroics undergoing order-disorder transformations accompanied by large change in the volume and as result in baric coefficient, $d T_{0} / d p=\delta V / \delta S$, are the most promising barocaloric materials. Important requirements for caloric materials are also their low cost and ecological tolerance.

It has recently been shown that ferrielectric $\left(\mathrm{NH}_{4}\right)_{2} \mathrm{SO}_{4}$ meets all of the above requirements [15. Due to significant values of $\Delta S=17 \mathrm{~J} / \mathrm{mol} \cdot \mathrm{K} \approx R \ln 8$ and $d T_{0} / d p=-45 \mathrm{~K} / \mathrm{GPa}$, rather large extensive and intensive BCE were observed in the region of the phase transition Pnam - Pna $2_{1}$ under low pressure. In accordance with Eq. 1, the negative baric coefficient associated with the negative value $(\partial V / \partial T)_{p}$ is the reason of the inverse $\mathrm{BCE}_{i n v}$ in $\left(\mathrm{NH}_{4}\right)_{2} \mathrm{SO}_{4}$ accompanied by increase/decrease in entropy/temperature under pressure increase. It was also found that large coefficient of the volume thermal expansion of the crystal lattice, $\beta_{L A T}$, can play an important role in formation of real $\mathrm{BCE}$ in material. Indeed, in the case of ammonium sulphate, large positive value $\beta_{L A T}=1.4 \times 10^{-4} \mathrm{~K}^{-1}$ leads to decrease in the inverse $\mathrm{BCE}_{\text {inv }}$ under pressure due to the appearance of the conventional contribution BCE $\left(\triangle S_{B C E}<0\right.$, 
$\left.\Delta T_{A D}>0\right)$ [15]. The conversion from $\mathrm{BCE}_{i n v}$ to $\mathrm{BCE}_{\text {conv }}$ was observed in a narrow temperature range. When pressure increases, the ratio between these values changes and at $p=0.25 \mathrm{GPa}$ is about $\mathrm{BCE}_{\text {conv }} / \mathrm{BCE}_{i n v}=0.15$. Thus, to get correct information on $\mathrm{BCE}$ in materials with large thermal expansion coefficient, it is necessary to take into account the effect of pressure on the lattice entropy. It is obvious that the magnitude of baric coefficient strongly effects on the maximum value of the intensive $\mathrm{BCE}$ [18. In this respect, it is interesting to analyze both BCE in material with anomalously large negative or positive $d T / d p$. From this point of view, another ferroelectric crystal, ammonium hydrogen sulphate, is very good example.

Indeed, $\mathrm{NH}_{4} \mathrm{HSO}_{4}$ undergoes two successive phase transitions $P 2_{1} / c \leftrightarrow$ $P c \leftrightarrow P 1$, of the strong second and first order at $T_{1}=271 \mathrm{~K}$ and $T_{2}=159 \mathrm{~K}$, respectively. One more difference is that anomaly of volumetric thermal expansion coefficient is positive at $T_{1}$ and negative at $T_{2}$ [19] which leads to $\mathrm{BCE}_{\text {conv }}$ and $\mathrm{BCE}_{i n v}$. Thus, the contribution from the thermal expansion of the crystal

40 lattice to both BCE will be also different. Despite the large difference in the entropy of the phase transitions $\left(\Delta S_{1}=1.2 \mathrm{~J} / \mathrm{mol} \cdot \mathrm{K}, \Delta S_{2}=7.6 \mathrm{~J} / \mathrm{mol} \cdot \mathrm{K}\right)$, one can suppose that $\mathrm{BCE}_{\text {conv }}$ at $T_{1}$ could be strongly increased due to rather large value of $\beta_{L A T}=2 \times 10^{-4} \mathrm{~K}^{-1}$ far from the phase transition points. In addition, like $\left(\mathrm{NH}_{4}\right)_{2} \mathrm{SO}_{4}$, ammonium hydrogen sulphate is also easy to prepare, cheap 45 and environmentally friendly.

In the present paper, we performed an analysis of extensive and intensive barocaloric efficiency of $\mathrm{NH}_{4} \mathrm{HSO}_{4}$ near both phase transformations based on methods developed by us earlier [18. For this aim, the dependencies of $\Delta T_{A D}(T, p)$ and $\Delta S_{B C E}(T, p)$ were determined using data on total and anomalous heat capacity [19], the $T-p$ phase diagram and the dependencies of entropy of the phase transitions on temperature and pressure. 


\section{Experimental details}

Powder samples of ammonium hydrogen sulphate were obtained by slow evaporation at $45^{\circ} \mathrm{C}$ from an aqueous solution containing equimolar quantities of high purity raw materials $\left(\mathrm{NH}_{4}\right)_{2} \mathrm{SO}_{4}$ and $\mathrm{H}_{2} \mathrm{SO}_{4}$.

The quality of samples used for the experiments was checked at room temperature using XRD, which revealed a monoclinic symmetry consistent with the space group $P 2_{1} / c(Z=8)$ suggested in Refs. [20, 21]. No additional phases were observed in the samples. Fig. 1 shows the results of Rietveld refinement.

${ }_{60}$ The unit cell parameters $a=24.770(6) \AA, b=4.611(1) \AA, c=14.871(4) \AA$, $\beta=89.70(1)$ grad are consistent with the values determined in Ref. 21].

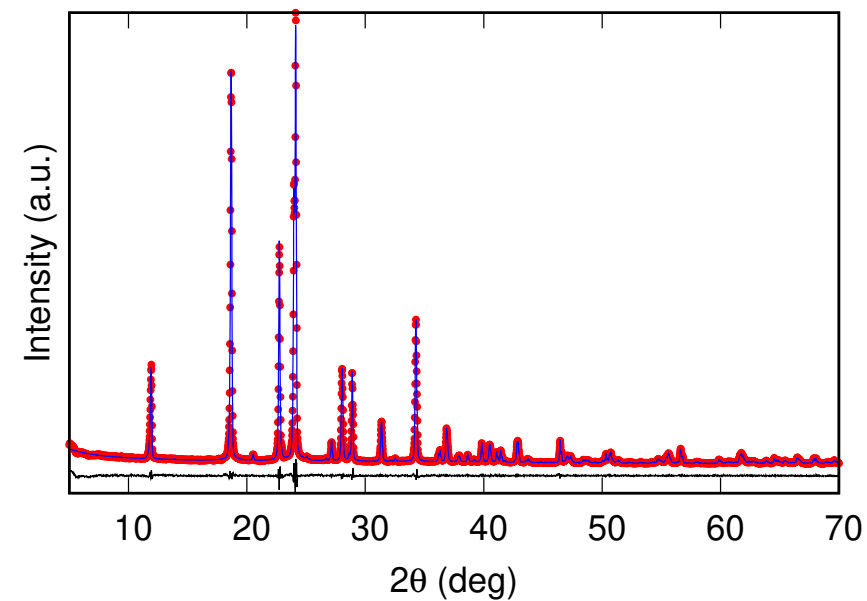

Figure 1: Difference Rietveld plot for $\mathrm{NH}_{4} \mathrm{HSO}_{4}$ at room temperature.

Quasi-ceramic samples of $\mathrm{NH}_{4} \mathrm{HSO}_{4}$ in the form of disks, approximately $1.0 \mathrm{~mm}$ thick and approximately $6 \mathrm{~mm}$ in diameter, were used for investigations. Because of the presence of ammonium ion in crystal, the heat treatments of ceramics were not performed. For dielectric measurements, electrodes on pellets were formed by conducting glue covered the opposite sides of the sample.

The effect of hydrostatic pressure on temperature and entropy of the phase transitions was studied using a piston-cylinder type vessel associated with a pressure multiplier. Pressure of up to $0.25 \mathrm{GPa}$ was generated using a mixture 
of silicon oil and pentane exhibiting optimal electrical and heat conductivity, solidification point and viscosity as the pressure-transmitting medium. Pressure and temperature were measured using a manganin resistive sensor and a copperconstantan thermocouple, with accuracies of about $\pm 10^{-3} \mathrm{GPa}$ and $\pm 0.3 \mathrm{~K}$ respectively.

The dependencies $T_{1}(p)$ and $T_{2}(p)$ were revealed, firstly, in experiments with differential thermal analysis (DTA) and, secondly, by measurements of the permittivity $\varepsilon$. In the former case, to detect anomalies of DTA-signal associated with the heat capacity anomalies, high-sensitive differential copper-germanium thermocouple was used. In the latter case, experiments were performed using an E7-20 immittance meter. To ensure the reliability of the results, the measurements were performed for both increasing and decreasing pressure cycles.

\section{Results and discussion}

At ambient pressure, the anomalies of DTA signal and $\varepsilon$ were detected at about $T_{1}=271.5 \pm 1.0 \mathrm{~K}$ and $T_{2}=160 \pm 2 \mathrm{~K}$ (Fig.2(a) and (b)), which agree well with values observed during measurements of the heat capacity [19]. Fig. 2(c) shows that an increase in pressure leads to linear increase and decrease in $T_{1}$ and $T_{2}$, respectively: $d T_{1} / d p=+90 \pm 15 \mathrm{~K} / \mathrm{GPa}$ and $d T_{2} / d p=-123 \pm 15 \mathrm{~K} / \mathrm{GPa}$ which are significantly higher than that for ammonium sulfate [15].

Due to the limited sensitivity of the DTA method, the area under the DTA 90 peak at $T_{2}$ represents a change in the enthalpy $\delta H_{2}$ (entropy $\delta S_{2}=\delta H_{2} / T_{2}$ ) jump at the first order phase transition $P c \leftrightarrow P 1$ in $\mathrm{NH}_{4} \mathrm{HSO}_{4}$. An increase in pressure is accompanied by a linear decrease in the value of $\delta S_{2}$ which reaches zero at $p \approx 0.17 \mathrm{GPa}$ (Fig. 2(d)) that can be considered as corresponding to the pressure of the tricritical point. On the other hand, it is unlikely that such a low pressure may affect the degree of disordering of structural elements in phases $P 2_{1} / c$ and $P c$, and as a result the total entropy change at the $P c \leftrightarrow P 1$ transformation, $\Delta S_{2}(p)=\delta S_{2}(p)+\Delta S_{2}^{*}(T, p)$, remains constant. Here $\Delta S_{2}^{*}(T, p)$ is the temperature- and pressure-dependent contribution. 

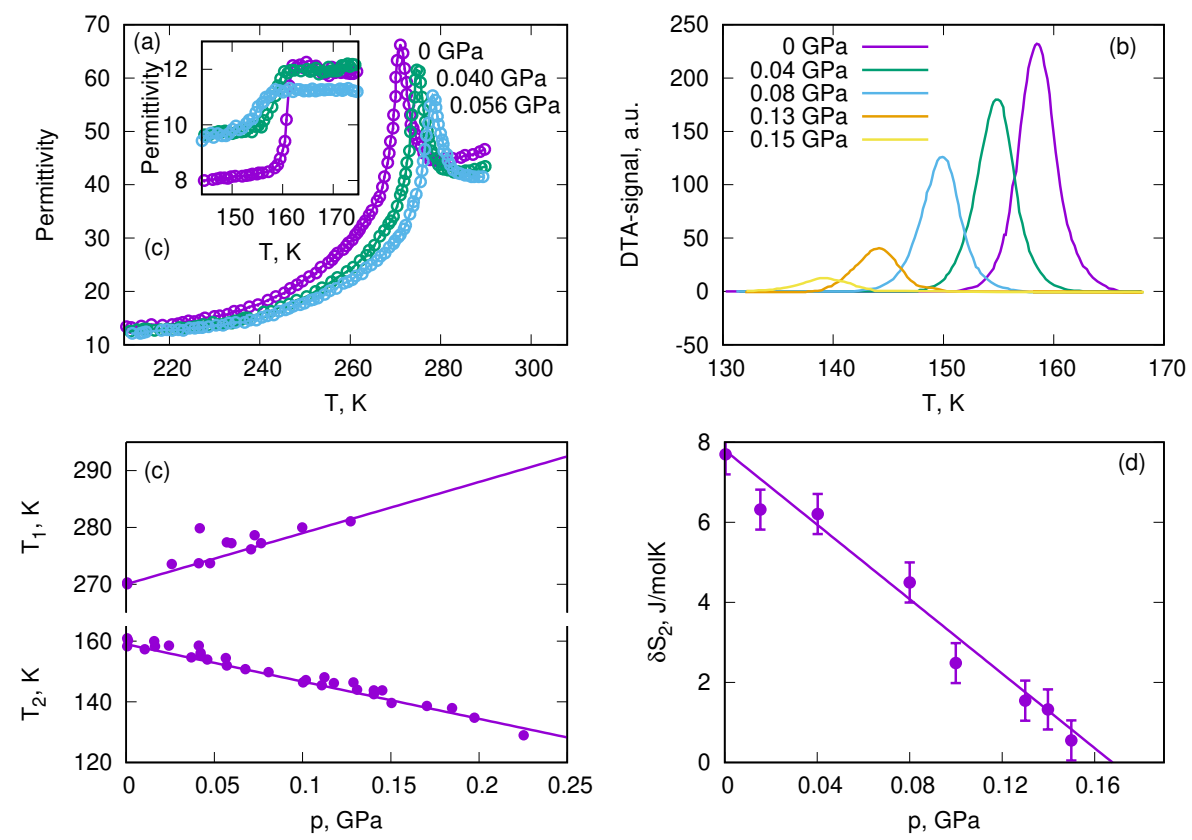

Figure 2: (a) Temperature dependencies of permittivity for $\mathrm{NH}_{4} \mathrm{HSO}_{4}$ around $T_{1}$ and $T_{2}$ and (b) anomalous component of the DTA signal near $T_{2}$ at different hydrostatic pressure. (c) Temperature - pressure phase diagram combining the results on the DTA signal and permittivity study. (d) Entropy jump $\delta S_{2}$ for the first-order transition in $\mathrm{NH}_{4} \mathrm{HSO}_{4}$ at different hydrostatic pressure.

The analysis of extensive and intensive $\mathrm{BCE}$ in $\mathrm{NH}_{4} \mathrm{HSO}_{4}$ was performed in three steps.
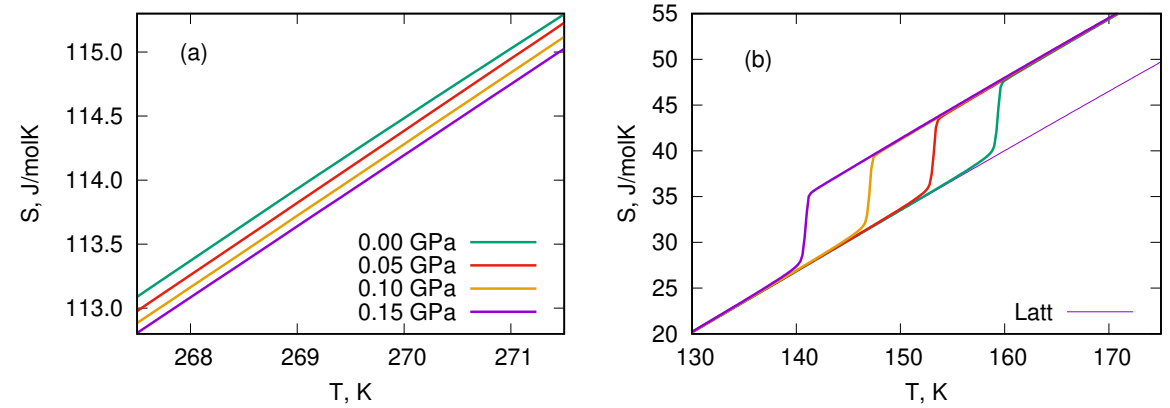

Figure 3: Temperature dependencies of total entropy of $\mathrm{NH}_{4} \mathrm{HSO}_{4}$ at different hydrostatic pressure near (a) $T_{1}$ and (b) $T_{2}$. 
At the first stage, the possible influence of pressure on the entropy of the crystal lattice $\Delta S_{L A T}$ was not taken into account. Fig. 3 demonstrates the temperature behavior of the lattice $S_{L A T}(T)-S_{L A T}(100 \mathrm{~K})=\int_{100}^{T}\left(C_{L A T} / T\right) d T$ and total $S=\int_{100}^{T}\left(C_{p} / T\right) d T$ entropies in the vicinities of $T_{1}$ and $T_{2}$. Temperature dependencies $S(T)$ under pressure were determined by summation of the lattice entropy $S_{L A T}$ and the anomalous contributions $\Delta S_{1}$ and $\Delta S_{2}$ shifted along the temperature scale in accordance with the sign of baric coefficients $d T_{1} / d p$ and $d T_{2} / d p$. The values and behavior of extensive BCE, $\triangle S_{B C E}$, at different pressure were determined from temperature dependencies of the total entropy as a difference $\Delta S_{B C E}=S(T, p)-S(T, p=0)$ at constant temperature (Fig. 4(a)). The temperature dependencies of the intensive BCE were revealed analyzing plots of $S(T, p)=S_{L A T}(T, p=0)+\Delta S(T, p)$ at constant entropy $S(T, p)=S\left(T+\Delta T_{A D}, p=0\right)$ (Fig. 4(b)). Large difference in BCE at $T_{1}$ and $T_{2}$ at the same pressure is the result of the different values of $\Delta S_{1}$ and $\Delta S_{2}$.

It is known [22] that if we neglect the contribution of the thermal expansion of the crystal lattice, the maximum values of both BCE are limited by the value of the entropy of the phase transition: $\left(\Delta S_{B C E}^{\max }\right)_{T 1}=\Delta S_{1}=-10 \mathrm{~J} / \mathrm{kg} \cdot \mathrm{K}$, $\left(\Delta T_{A D}^{\max }\right)_{T 1}=2 \mathrm{~K} ;\left(\Delta S_{B C E}^{\max }\right)_{T 2}=\Delta S_{2}=68 \mathrm{~J} / \mathrm{kg} \cdot \mathrm{K},\left(\Delta T_{A D}^{\max }\right)_{T 2}=12 \mathrm{~K}$. However undoubtedly important is the ability to realize in the material maximum values of both BCE at low pressure.

Estimates made using the following relation $p_{\min }=T \Delta S /\left(C_{L A T} d T / d p\right)$, valid for phase transitions of the first order [9], show that the value $\left(\triangle S_{B C E}^{\max }\right)_{T 2}$ can be implemented in $\mathrm{NH}_{4} \mathrm{HSO}_{4}$ by rather insignificant pressure $0.1 \mathrm{GPa}$. In fact, the rather large value $\left(\Delta S_{B C E}\right)_{T 2}=0.95\left(\Delta S_{B C E}^{\max }\right)_{T 2}$ can be achieved even at much lower pressure, $p \approx 0.02 \mathrm{GPa}$ (Fig. 4(a)). However, intensive BCE, which also depends on the $d S_{L A T} / d T$ derivative, is characterized by lower increase rate under pressure and reaches $\left(\Delta T_{A D}^{\max }\right)_{T 2}$ at about $0.13 \mathrm{GPa}$ (Fig. 4(b)). As to the second order phase transition, at pressure 0.1 GPa extensive and intensive effects reach only about $18 \%$ of $\left(\Delta S_{B C E}^{\max }\right)_{T 1}$ and $\left(\Delta T_{A D}^{\max }\right)_{T 1}$.

At the second stage, effect of the lattice entropy change under pressure on $\mathrm{BCE}$ in $\mathrm{NH}_{4} \mathrm{HSO}_{4}$ was studied. This contribution can be evaluated using 

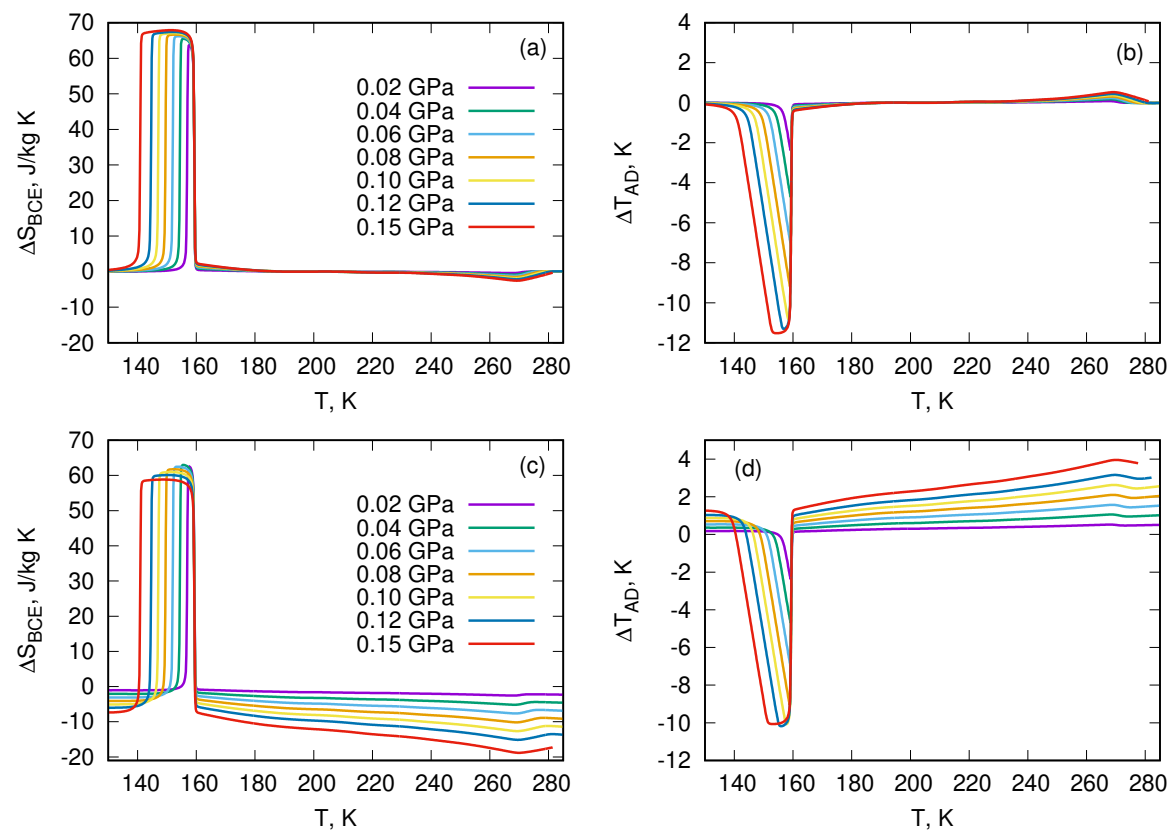

Figure 4: (a) Barocaloric entropy and (b) adiabatic temperature changes at different hydrostatic pressure in a wide temperature range determined without taking into account the effect of thermal expansion of the crystal lattice. Effect of thermal expansion of the crystal lattice on (c) $\Delta S_{B C E}\left(\mathrm{~d} \Delta T_{A D}\right)$

Maxwell relation $\left(\partial S_{L A T} / \partial p\right)_{T}=-(\partial V / \partial T)_{p}$

$$
\Delta S_{L A T}(T, p)=-\int_{0}^{p}(\partial V / \partial T)_{p} d p \approx-V_{m} \beta_{L A T}(T) p .
$$

130

Here $\left.V_{m}=6.17 \times 10^{-5} \mathrm{~m}^{3} / \mathrm{mol}\right)$ is the molar volume.

Taken into account the data of the thermal expansion study of the related $\left(\mathrm{NH}_{4}\right)_{2} \mathrm{SO}_{4}\left[15\right.$, it was suggested that the values of $\beta_{L A T}$ and $V_{m}$ of $\mathrm{NH}_{4} \mathrm{HSO}_{4}$ are also weakly depend on the pressure. Lattice contribution was determined from the results of dilatometric study of $\mathrm{NH}_{4} \mathrm{HSO}_{4}[19$.

Fig. 4(c) and (d) demonstrate that due to the same sign of both derivatives, $(\partial V / \partial T)_{T 1}$ and $\left(\partial V_{L A T} / \partial T\right)$, there is a strong increase in $\mathrm{BCE}_{c o n v}$. At $p=0.15 \mathrm{GPa}$, the values $\left(\Delta S_{B C E}\right)_{T 1}=-18.8 \pm 1.5 \mathrm{~J} / \mathrm{kg} \cdot \mathrm{K}$ and $\left(\Delta T_{A D}\right)_{T 1}=$ $4.0 \pm 0.2 \mathrm{~K}$ are about one and a half times higher than even the maximum values considered above as associated with the phase transition entropy $\Delta S_{1}$. 


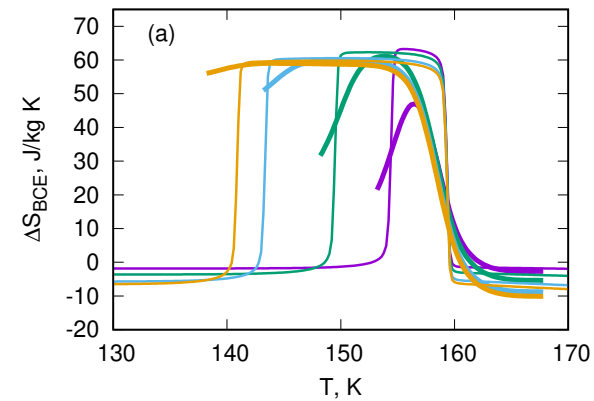
predominant. $1.5 \pm 0.1 \mathrm{~K}$.

Thus, contributions of $\left(\Delta S_{B C E}^{L A T}\right)_{T 1}$ and $\left(\Delta T_{A D}^{L A T}\right)_{T 1}$ to the full BCE at $T_{1}$ are

On the other hand, at the same pressure, $\mathrm{BCE}_{i n v}$ associated with the phase transition $P c-P 1\left((\partial V / \partial T)_{T 2}<0\right)$, is reduced to the magnitudes $\left(\Delta S_{B C E}\right)_{T 2}=$ $59 \pm 5 \mathrm{~J} / \mathrm{kg} \cdot \mathrm{K},\left(\Delta T_{A D}\right)_{T 2}=-10.0 \pm 0.8 \mathrm{~K}$ by $\mathrm{BCE}_{\text {conv }}^{L A T}$ arising in accordance with Eq. 2. $\left(\partial V_{L A T} / \partial T\right)>0:\left(\Delta S_{B C E}^{L A T}\right)_{T 2}=-9.0 \pm 0.7 \mathrm{~J} / \mathrm{kg} \cdot \mathrm{K},\left(\Delta T_{A D}^{L A T}\right)_{T 2}=$

At last, at the third stage, we determine the behavior of extensive and intensive $\mathrm{BCE}$ at $T_{2}$ taken into account the peculiarities of experiments with DTA under pressure. As it was discussed above, increase in pressure strongly decreases the entropy jump $\delta S_{2}$ at the first order phase transition $P c-P 1$ (Fig. 2(b) and (d)), while the total entropy change $\Delta S_{2}$ remains constant. Analyzing the dependencies $S(T, p)$ using both the DTA data under pressure and the effect of the lattice expansion, barocaloric parameters at $T_{2}$ are determined and presented in Fig. 5 in comparison with the data obtained in the second stage.

55 One can see that in this case the magnitudes of $\left(\Delta S_{B C E}^{\max }\right)_{T 2}$ and $\left(\Delta T_{A D}^{\max }\right)_{T 2}$ are realized at almost the same low pressure which was determined in the second stage (Fig. 4(c) and (d)). The main difference was observed in the form of peaks $\left(\Delta S_{B C E}\right)_{T 2}(T)$ and $\left(\Delta T_{A D}\right)_{T 2}(T)$ which is due to peculiarities of the processes of measuring the heat capacity by methods of adiabatic calorimeter and DTA.

Figure 5: (a) Barocaloric entropy and (b) adiabatic temperature changes at different hydrostatic pressure determined using DTA data on a jump of the entropy (thick lines) and data presented in Fig, 4(c) and (d) (thin lines). 
Returning to the results of the BCE study at $T_{1}$, one can confidently argue that it would be interesting and useful to investigate the influence of the thermal expansion of the crystal lattice on conventional BCE in ferroelectrics characterized by large $\beta_{L A T}$ and undergoing order-disorder transformation with the positive baric coefficient.

\section{Conclusion}

This paper demonstrates $\mathrm{BCE}$ in $\mathrm{NH}_{4} \mathrm{HSO}_{4}$ undergoing two successive ferroelectric phase transitions of displacive and order-disorder type at $T_{1}$ and at $T_{2}$, respectively. Both transformations are characterized by large baric coefficients of different sign. Hydrostatic pressure strongly decreases the entropy jump at $T_{2}$ which reaches zero at a pressure of $0.17 \mathrm{GPa}$. Very low pressure is needed to realize the maximum values of the extensive and intensive inverse BCE. Large thermal expansion of the crystal lattice leads to two very important points. Firstly, the conventional BCE can be greatly increased to values much higher than the magnitudes corresponding to the entropy of the phase transition. Secondly, in the case of the negative baric coefficient, a conversion from the inverse to conventional $\mathrm{BCE}$ can be realized by low pressure in the narrow temperature range.

\section{References}

[1] K. A. GschneidnerJr, V. K. Pecharsky, A. O. Tsokol, Recent developments in magnetocaloric materials, Rep. Prog. Phys. 68 (6) (2005) 1479-1539. doi:10.1088/0034-4885/68/6/r04.

[2] M. Valant, Electrocaloric materials for future solid-state refrigeration technologies, Prog. Mater. Sci. 57 (6) (2012) 980 - 1009. doi:10.1016/j. pmatsci.2012.02.001.

[3] X. Moya, S. Kar-Narayan, N. D. Mathur, Caloric materials near ferroic phase transitions, Nat. Mater. 13 (2014) 439-450. doi:10.1038/nmat3951 
[4] H. Khassaf, T. Patel, R. J. Hebert, S. P. Alpay, Flexocaloric response of epitaxial ferroelectric films, J. Appl. Phys. 123 (2) (2018) 024102. doi: $10.1063 / 1.5009121$

[5] U. Tomc, J. Tušek, A. Kitanovski, A. Poredoš, A new magnetocaloric refrigeration principle with solid-state thermoelectric thermal diodes, Appl. Thermal Engineering 58 (1) (2013) 1-10. doi:10.1016/j. applthermaleng.2013.03.063.

[6] U. Plaznik, M. Vrabelj, Z. Kutnjak, B. Malič, A. Poredoš, A. Kitanovski, Electrocaloric cooling: The importance of electric-energy recovery and heat

口 regeneration, EPL (Europhysics Letters) 111 (5) (2015) 57009. doi:10. 1209/0295-5075/111/57009.

[7] A. Kitanovski, U. Plaznik, U. Tomc, A. Poredo, Present and future caloric refrigeration and heat-pump technologies, Int. J. Refrigeration 57 (2015) 288 - 298. doi:10.1016/j.ijrefrig.2015.06.008.

[8] N. Michaelis, F. Welsch, S.-M. Kirsch, M. Schmidt, S. Seelecke, A. Schütze, Experimental parameter identification for elastocaloric air cooling, Int. J. Refrigeration 100 (2019) 167 - 174. doi:10.1016/j.ijrefrig.2019.01. 006.

[9] M. Gorev, E. Bogdanov, I. Flerov, T-p phase diagrams and the barocaloric effect in materials with successive phase transitions, J. Phys. D: Appl. Phys. 50 (38) (2017) 384002. doi:10.1088/1361-6463/aa8025

[10] M. Gorev, E. Bogdanov, I. Flerov, Conventional and inverse barocaloric effects around triple points in ferroelastics $\left(\mathrm{NH}_{4}\right)_{3} \mathrm{NbOF}_{6}$ and $\left(\mathrm{NH}_{4}\right)_{3} \mathrm{TiOF}_{5}$, 210 ㄴ Scripta Materialia 139 (2017) 53-57. doi:10.1016/j.scriptamat.2017. 06.022

[11] E. Mikhaleva, I. Flerov, A. Kartashev, M. Gorev, A. Cherepakhin, K. Sablina, N. Mikhashenok, N. Volkov, A. Shabanov, Caloric effects and phase transitions in ferromagnetic-ferroelectric composites $\mathrm{xLa}_{0} \cdot{ }_{7} \mathrm{~Pb}_{0}$. 
${ }_{3} \mathrm{MnO}_{3}-(1-\mathrm{x}) \mathrm{PbTiO}_{3}$, J. Mater. Res. 28 (24) (2013) 3322-3331. doi: $10.1557 / \mathrm{jmr} .2013 .360$.

[12] K. Alex Müller, F. Fauth, S. Fischer, M. Koch, A. Furrer, P. Lacorre, Cooling by adiabatic pressure application in $\mathrm{Pr}_{1}-\mathrm{xLaxNiO} \mathrm{N}_{3}$, Appl. Phys. Letters 73 (8) (1998) 1056-1058. doi:10.1063/1.122083.

[13] T. Strässle, A. Furrer, Z. Hossain, C. Geibel, Magnetic cooling by the application of external pressure in rare-earth compounds, Phys. Rev. B 67 (2003) 054407. doi:10.1103/PhysRevB.67.054407.

[14] N. A. de Oliveira, Barocaloric effect and the pressure induced solid state refrigerator, J. Appl. Phys. 109 (5) (2011) 053515. doi:10.1063/1.3556740

[15] P. Lloveras, E. Stern-Taulats, M. Barrio, J.-L. Tamarit, S. Crossley, W. Li, V. Pomjakushin, A. Planes, L. Manosa, N. D. Mathur, X. Moya, Giant barocaloric effects at low pressure in ferrielectric ammonium sulphate, Nat. Commun. 6 (2015) 8801. doi:10.1038/ncomms9801.

[16] H. Khassaf, T. Patel, S. P. Alpay, Combined intrinsic elastocaloric and electrocaloric properties of ferroelectrics, J. Appl. Phys. 121 (14) (2017) 144102. doi:10.1063/1.4980098.

[17] Y. Liu, J. Wei, P.-E. Janolin, I. C. Infante, X. Lou, B. Dkhil, Giant roomtemperature barocaloric effect and pressure-mediated electrocaloric effect

q in $\mathrm{BaTiO}_{3}$ single crystal, Appl. Phys. Letters 104 (16) (2014) 162904. doi: $10.1063 / 1.4873162$

[18] M. V. Gorev, I. N. Flerov, E. V. Bogdanov, V. N. Voronov, N. M. Laptash, Barocaloric effect near the structural phase transition in the घ $\mathrm{Rb}_{2} \mathrm{KTiOF}_{5}$ oxyfluoride, Phys. Solid State 52 (2) (2010) 377-383. doi: $10.1134 / \mathrm{S} 1063783410020253$

[19] E. Mikhaleva, I. Flerov, A. Kartashev, M. Gorev, E. Bogdanov, V. Bondarev, Thermal, dielectric and barocaloric properties of $\mathrm{NH}_{4} \mathrm{HSO}_{4}$ crystal- 
lized from an aqueous solution and the melt, Solid State Sciences 67 (2017)

1-7. doi:10.1016/j.solidstatesciences.2017.03.004.

[20] R. Pepinsky, K. Vedam, S. Hoshino, Y. Okaya, Ammonium hydrogen sulfate: A new ferroelectric with low coercive field, Phys. Rev. 111 (1958) 1508-1510. doi:10.1103/PhysRev.111.1508.

[21] D. Swain, V. S. Bhadram, P. Chowdhury, C. Narayana, Raman and x-ray investigations of ferroelectric phase transition in $\mathrm{NH}_{4} \mathrm{HSO}_{4}$, J. Phys. Chem. A 116 (2012) 223-230. doi:10.1021/jp2075868

[22] R. Pirc, Z. Kutnjak, R. Blinc, Q. M. Zhang, Upper bounds on the electrocaloric effect in polar solids, Appl. Phys. Letters 98 (2) (2011) 021909. doi:10.1063/1.3543628. 


\title{
Conventional and inverse barocaloric effects in ferroelectric $\mathrm{NH}_{4} \mathrm{HSO}_{4}$
}

\author{
Mikhail V. Gorev ${ }^{\mathrm{a}, \mathrm{b}}$, Ekaterina A. Mikhaleva, ${ }^{\mathrm{a}, \mathrm{b}}$, Igor N. Flerov ${ }^{\mathrm{a}, \mathrm{b}, *}$, Evgeniy \\ V. Bogdanov ${ }^{\mathrm{a}, \mathrm{c}}$ \\ ${ }^{a}$ Kirensky Institute of Physics, Federal Research Center KSC SB RAS, Krasnoyarsk, Russia \\ ${ }^{b}$ Institute of Engineering Physics and Radioelectronics, Siberian Federal University, \\ Krasnoyarsk, Russia \\ ${ }^{c}$ Institute of Engineering Systems and Energy, Krasnoyarsk State Agrarian University, \\ 660049 Krasnoyarsk, Russia
}

\begin{abstract}
In this study, the conventional and inverse barocaloric effects (BCE) in ferroelectric $\mathrm{NH}_{4} \mathrm{HSO}_{4}$ are reported. Maximum extensive and intensive $\mathrm{BCE}$ near order-disorder phase transition can be achieved at low pressure $p \leq 0.1 \mathrm{GPa}$. Large thermal expansion of the crystal lattice plays a very important role in the developing conventional BCE and conversation between BCE of different sign in the narrow temperature range.
\end{abstract}

Keywords: Polymorphic phase transformation, Phase diagram,

Order-disorder phenomena, Entropy, Barocaloric effect

PACS: 62.50.-p, 65.40.-b, 81.30.-t

\section{Introduction}

In recent years, much attention is paid to caloric effects (CE) in solids, particularly in ferroics, associated with the reversible change in the temperature, $\Delta T_{A D}$, or entropy, $\Delta S_{C E}$, under variation of the external field in adiabatic and isothermal conditions, respectively [1, 2, 3, 4]. One of the main reasons for this interest is related to the possibility to use the materials showing large CE's

\footnotetext{
* Corresponding author

Email addresses: gorev@iph.krasn.ru (Mikhail V. Gorev), katerina@iph.krasn.ru (Ekaterina A. Mikhaleva), flerov@iph.krasn.ru (Igor N. Flerov), evbogdanov@iph.krasn.ru (Evgeniy V. Bogdanov)
}

Preprint submitted to Journal of Alloys and Compounds

July 22, 2019 
as solid state refrigerants in alternative cooling cycles [5, 6, 7, 8]. Among the CE's of different physical nature, the barocaloric effect (BCE) is distinguished by a serious advantage associated with its universality. Indeed, both extensive $\Delta S_{B C E}$ and intensive $\Delta T_{A D}$ barocaloric parameters strongly depend on the volume thermal expansion $(\partial V / \partial T)_{p}$ which very often shows large change near the temperature of any phase transitions: ferroelectric, ferroelastic, ferromagnetic

$$
\Delta S_{B C E}=-\int_{0}^{p}\left(\frac{\partial V}{\partial T}\right)_{p} d p, \Delta T_{A D}=-\frac{T}{C_{p}} \Delta S_{B C E},
$$

where $C_{p}$ is the heat capacity.

The most intensively, BCE was studied in materials undergoing ferroelastic [9, 10] and ferromagnetic [11, 12, 13, 14, phase transitions. As to the ferro5 electrics, their barocaloric efficiency was investigated only sporadically [11, 15, 16, 17. It is known that the values and behavior of the BCE depend on the behavior and change in the entropy $\Delta S$ of the phase transition as well as on the sensitivity of the phase transition temperature to hydrostatic pressure [9, 10]. Thus, ferroics undergoing order-disorder transformations accompanied by large change in the volume and as result in baric coefficient, $d T_{0} / d p=\delta V / \delta S$, are the most promising barocaloric materials. Important requirements for caloric materials are also their low cost and ecological tolerance.

It has recently been shown that ferrielectric $\left(\mathrm{NH}_{4}\right)_{2} \mathrm{SO}_{4}$ meets all of the above requirements [15]. Due to significant values of $\Delta S=17 \mathrm{~J} / \mathrm{mol} \cdot \mathrm{K} \approx R \ln 8$ and $d T_{0} / d p=-45 \mathrm{~K} / \mathrm{GPa}$, rather large extensive and intensive BCE were observed in the region of the phase transition Pnam - Pna2 $2_{1}$ under low pressure. In accordance with Eq. 1, the negative baric coefficient associated with the negative value $(\partial V / \partial T)_{p}$ is the reason of the inverse $\mathrm{BCE}_{\text {inv }}$ in $\left(\mathrm{NH}_{4}\right)_{2} \mathrm{SO}_{4}$ accompanied by increase/decrease in entropy/temperature under pressure increase. It was also found that large coefficient of the volume thermal expansion of the crystal lattice, $\beta_{L A T}$, can play an important role in formation of real $\mathrm{BCE}$ in material. Indeed, in the case of ammonium sulphate, large positive value $\beta_{L A T}=1.4 \times 10^{-4} \mathrm{~K}^{-1}$ leads to decrease in the inverse $\mathrm{BCE}_{i n v}$ under pressure due to the appearance of the conventional contribution BCE $\left(\triangle S_{B C E}<0\right.$, 
$\left.\Delta T_{A D}>0\right)$ [15]. The conversion from $\mathrm{BCE}_{i n v}$ to $\mathrm{BCE}_{\text {conv }}$ was observed in a narrow temperature range. When pressure increases, the ratio between these values changes and at $p=0.25 \mathrm{GPa}$ is about $\mathrm{BCE}_{\text {conv }} / \mathrm{BCE}_{i n v}=0.15$. Thus, to get correct information on $\mathrm{BCE}$ in materials with large thermal expansion coefficient, it is necessary to take into account the effect of pressure on the lattice entropy. It is obvious that the magnitude of baric coefficient strongly effects on the maximum value of the intensive $\mathrm{BCE}$ [18. In this respect, it is interesting to analyze both BCE in material with anomalously large negative or positive $d T / d p$. From this point of view, another ferroelectric crystal, ammonium hydrogen sulphate, is very good example.

Indeed, $\mathrm{NH}_{4} \mathrm{HSO}_{4}$ undergoes two successive phase transitions $P 2_{1} / c \leftrightarrow$ $P c \leftrightarrow P 1$, of the strong second and first order at $T_{1}=271 \mathrm{~K}$ and $T_{2}=159 \mathrm{~K}$, respectively. One more difference is that anomaly of volumetric thermal expansion coefficient is positive at $T_{1}$ and negative at $T_{2}$ [19] which leads to $\mathrm{BCE}_{\text {conv }}$ and $\mathrm{BCE}_{i n v}$. Thus, the contribution from the thermal expansion of the crystal

40 lattice to both BCE will be also different. Despite the large difference in the entropy of the phase transitions $\left(\Delta S_{1}=1.2 \mathrm{~J} / \mathrm{mol} \cdot \mathrm{K}, \Delta S_{2}=7.6 \mathrm{~J} / \mathrm{mol} \cdot \mathrm{K}\right)$, one can suppose that $\mathrm{BCE}_{\text {conv }}$ at $T_{1}$ could be strongly increased due to rather large value of $\beta_{L A T}=2 \times 10^{-4} \mathrm{~K}^{-1}$ far from the phase transition points. In addition, like $\left(\mathrm{NH}_{4}\right)_{2} \mathrm{SO}_{4}$, ammonium hydrogen sulphate is also easy to prepare, cheap 45 and environmentally friendly.

In the present paper, we performed an analysis of extensive and intensive barocaloric efficiency of $\mathrm{NH}_{4} \mathrm{HSO}_{4}$ near both phase transformations based on methods developed by us earlier [18. For this aim, the dependencies of $\Delta T_{A D}(T, p)$ and $\Delta S_{B C E}(T, p)$ were determined using data on total and anomalous heat capacity [19], the $T-p$ phase diagram and the dependencies of entropy of the phase transitions on temperature and pressure. 


\section{Experimental details}

Powder samples of ammonium hydrogen sulphate were obtained by slow evaporation at $45^{\circ} \mathrm{C}$ from an aqueous solution containing equimolar quantities of high purity raw materials $\left(\mathrm{NH}_{4}\right)_{2} \mathrm{SO}_{4}$ and $\mathrm{H}_{2} \mathrm{SO}_{4}$.

The quality of samples used for the experiments was checked at room temperature using XRD, which revealed a monoclinic symmetry consistent with the space group $P 2_{1} / c(Z=8)$ suggested in Refs. [20, 21]. No additional phases were observed in the samples. Fig. 1 shows the results of Rietveld refinement $\left(R_{w p}=6.04, R_{p}=4.23, \chi^{2}=2.06\right)$. The unit cell parameters $a=24.770(6) \AA$, $b=4.611(1) \AA, c=14.871(4) \AA, \beta=89.70(1) \operatorname{grad}$ are consistent with the values determined in Ref. [21].

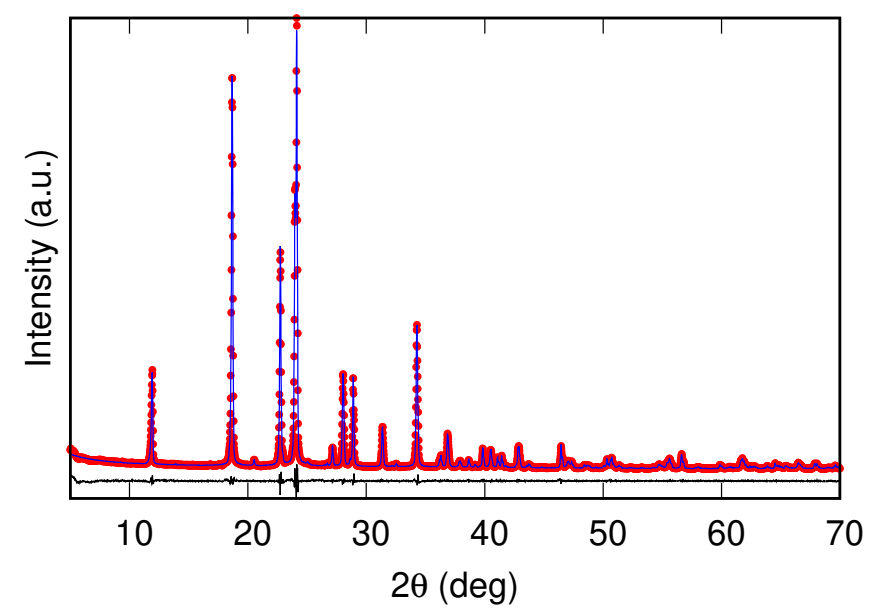

Figure 1: Difference Rietveld plot for $\mathrm{NH}_{4} \mathrm{HSO}_{4}$ at room temperature.

Quasi-ceramic samples of $\mathrm{NH}_{4} \mathrm{HSO}_{4}$ in the form of disks, approximately $1.0 \mathrm{~mm}$ thick and approximately $6 \mathrm{~mm}$ in diameter, were used for investigations. Because of the presence of ammonium ion in crystal, the heat treatments of ceramics were not performed. For dielectric measurements, electrodes on pellets were formed by conducting glue covered the opposite sides of the sample.

The effect of hydrostatic pressure on temperature and entropy of the phase transitions was studied using a piston-cylinder type vessel associated with a 
pressure multiplier. Pressure of up to $0.25 \mathrm{GPa}$ was generated using a mixture of silicon oil and pentane exhibiting optimal electrical and heat conductivity, solidification point and viscosity as the pressure-transmitting medium. Pressure and temperature were measured using a manganin resistive sensor and a copperconstantan thermocouple, with accuracies of about $\pm 10^{-3} \mathrm{GPa}$ and $\pm 0.3 \mathrm{~K}$ 75 respectively.

The dependencies $T_{1}(p)$ and $T_{2}(p)$ were revealed, firstly, in experiments with differential thermal analysis (DTA) and, secondly, by measurements of the permittivity $\varepsilon$. In the former case, to detect anomalies of DTA-signal associated with the heat capacity anomalies, high-sensitive differential copper-germanium thermocouple was used. In the latter case, experiments were performed using an E7-20 immittance meter. To ensure the reliability of the results, the measurements were performed for both increasing and decreasing pressure cycles.

\section{Results and discussion}

At ambient pressure, the anomalies of DTA signal and $\varepsilon$ were detected at about $T_{1}=271.5 \pm 1.0 \mathrm{~K}$ and $T_{2}=160 \pm 2 \mathrm{~K}$ (Fig.2(a) and (b)), which agree well with values observed during measurements of the heat capacity [19]. Fig. 2(c) shows that an increase in pressure leads to linear increase and decrease in $T_{1}$ and $T_{2}$, respectively: $d T_{1} / d p=+90 \pm 15 \mathrm{~K} / \mathrm{GPa}$ and $d T_{2} / d p=-123 \pm 15 \mathrm{~K} / \mathrm{GPa}$ which are significantly higher than that for ammonium sulfate [15].

Due to the limited sensitivity of the DTA method, the area under the DTA peak at $T_{2}$ represents a change in the enthalpy $\delta H_{2}$ (entropy $\left.\delta S_{2}=\delta H_{2} / T_{2}\right)$ jump at the first order phase transition $P c \leftrightarrow P 1$ in $\mathrm{NH}_{4} \mathrm{HSO}_{4}$. An increase in pressure is accompanied by a linear decrease in the value of $\delta S_{2}$ which reaches zero at $p \approx 0.17 \mathrm{GPa}$ (Fig. 2(d)) that can be considered as corresponding to the pressure of the tricritical point. On the other hand, it is unlikely that such a low pressure may affect the degree of disordering of structural elements in phases $P 2_{1} / c$ and $P c$, and as a result the total entropy change at the $P c \leftrightarrow P 1$ transformation, $\Delta S_{2}(p)=\delta S_{2}(p)+\Delta S_{2}^{*}(T, p)$, remains constant. Here $\Delta S_{2}^{*}(T, p)$ is 

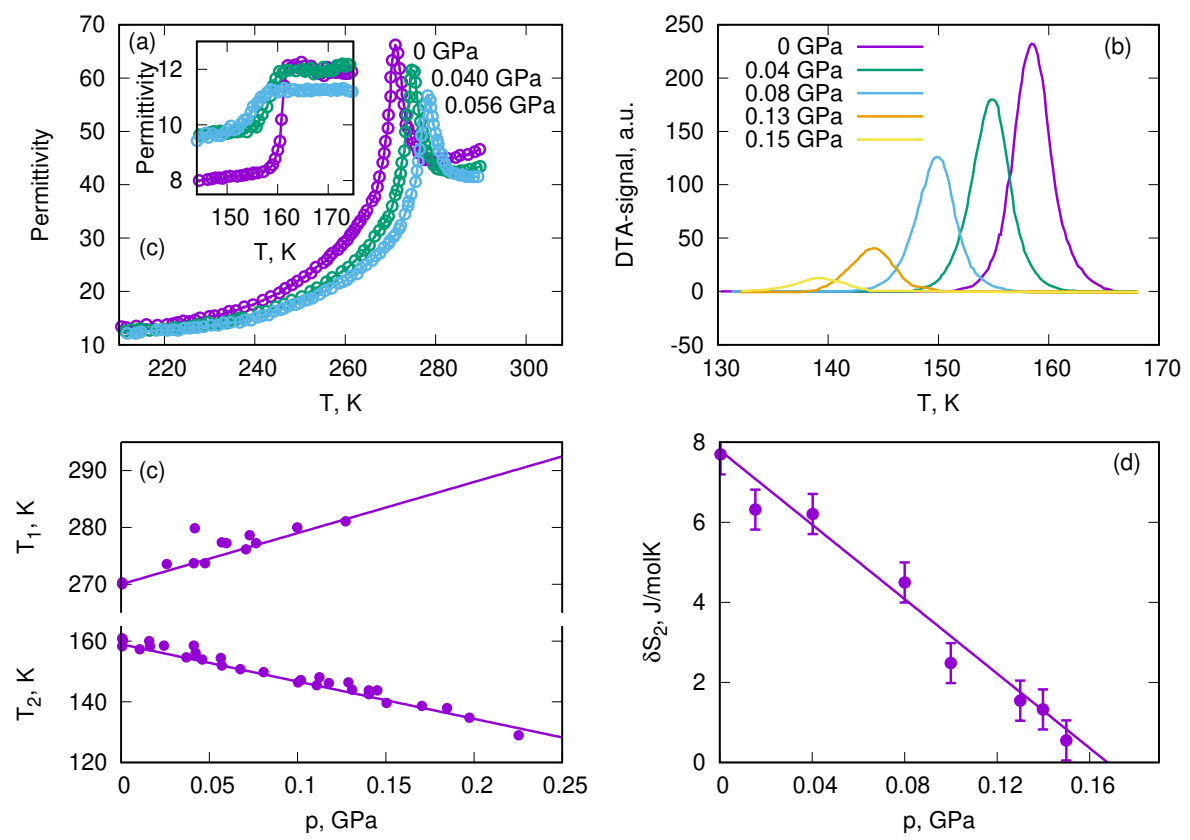

Figure 2: (a) Temperature dependencies of permittivity for $\mathrm{NH}_{4} \mathrm{HSO}_{4}$ around $T_{1}$ and $T_{2}$ and (b) anomalous component of the DTA signal near $T_{2}$ at different hydrostatic pressure. (c) Temperature - pressure phase diagram combining the results on the DTA signal and permittivity study. (d) Entropy jump $\delta S_{2}$ for the first-order transition in $\mathrm{NH}_{4} \mathrm{HSO}_{4}$ at different hydrostatic pressure.

the temperature- and pressure-dependent contribution.

The analysis of extensive and intensive $\mathrm{BCE}$ in $\mathrm{NH}_{4} \mathrm{HSO}_{4}$ was performed in three steps.

At the first stage, the possible influence of pressure on the entropy of the crystal lattice $\Delta S_{L A T}$ was not taken into account. Fig. 3 demonstrates the temperature behavior of the lattice $S_{L A T}(T)-S_{L A T}(100 \mathrm{~K})=\int_{100}^{T}\left(C_{L A T} / T\right) d T$ and total $S=\int_{100}^{T}\left(C_{p} / T\right) d T$ entropies in the vicinities of $T_{1}$ and $T_{2}$. Temperature dependencies $S(T)$ under pressure were determined by summation of the lattice entropy $S_{L A T}$ and the anomalous contributions $\Delta S_{1}$ and $\Delta S_{2}$ shifted along the temperature scale in accordance with the sign of baric coefficients $d T_{1} / d p$ and $d T_{2} / d p$. The values and behavior of extensive BCE, $\triangle S_{B C E}$, at different pressure were determined from temperature dependencies of the total 

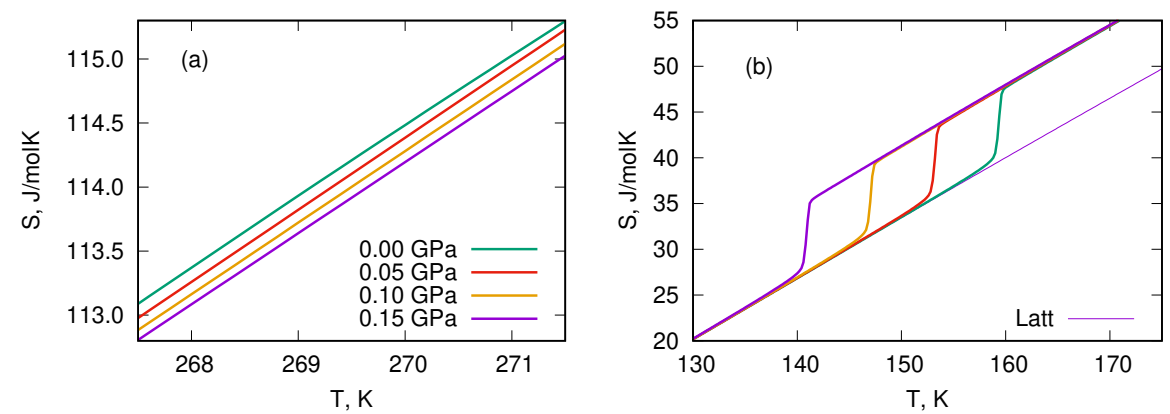

Figure 3: Temperature dependencies of total entropy of $\mathrm{NH}_{4} \mathrm{HSO}_{4}$ at different hydrostatic pressure near (a) $T_{1}$ and (b) $T_{2}$.

entropy as a difference $\Delta S_{B C E}=S(T, p)-S(T, p=0)$ at constant temperature (Fig. $4(\mathrm{a})$ ). The temperature dependencies of the intensive BCE were revealed analyzing plots of $S(T, p)=S_{L A T}(T, p=0)+\Delta S(T, p)$ at constant entropy $S(T, p)=S\left(T+\Delta T_{A D}, p=0\right)$ (Fig. $\left.4(\mathrm{~b})\right)$. Large difference in BCE at $T_{1}$ and $T_{2}$ at the same pressure is the result of the different values of $\Delta S_{1}$ and $\Delta S_{2}$.

It is known 22 that if we neglect the contribution of the thermal expansion of the crystal lattice, the maximum values of both $\mathrm{BCE}$ are limited by the value of the entropy of the phase transition: $\left(\triangle S_{B C E}^{\max }\right)_{T 1}=\Delta S_{1}=-10 \mathrm{~J} / \mathrm{kg} \cdot \mathrm{K}$, $\left(\Delta T_{A D}^{\max }\right)_{T 1}=2 \mathrm{~K} ;\left(\Delta S_{B C E}^{\max }\right)_{T 2}=\Delta S_{2}=68 \mathrm{~J} / \mathrm{kg} \cdot \mathrm{K},\left(\Delta T_{A D}^{\max }\right)_{T 2}=12 \mathrm{~K}$. However, undoubtedly important is the ability to realize in the material maximum values of both BCE at low pressure.

Estimates made using the following relation $p_{\min }=T \Delta S /\left(C_{L A T} d T / d p\right)$, valid for phase transitions of the first order $[9]$, show that the value $\left(\triangle S_{B C E}^{\max }\right)_{T 2}$ can be implemented in $\mathrm{NH}_{4} \mathrm{HSO}_{4}$ by rather insignificant pressure $0.1 \mathrm{GPa}$. In fact, the rather large value $\left(\Delta S_{B C E}\right)_{T 2}=0.95\left(\Delta S_{B C E}^{\max }\right)_{T 2}$ can be achieved even at much lower pressure, $p \approx 0.02 \mathrm{GPa}$ (Fig. 4(a)). However, intensive $\mathrm{BCE}$, which also depends on the $d S_{L A T} / d T$ derivative, is characterized by lower increase rate under pressure and reaches $\left(\Delta T_{A D}^{\max }\right)_{T 2}$ at about $0.13 \mathrm{GPa}$ (Fig. $4(\mathrm{~b})$ ). As to the second order phase transition, at pressure 0.1 GPa exten130 sive and intensive effects reach only about $18 \%$ of $\left(\Delta S_{B C E}^{\max }\right)_{T 1}$ and $\left(\Delta T_{A D}^{\max }\right)_{T 1}$.

At the second stage, effect of the lattice entropy change under pressure 

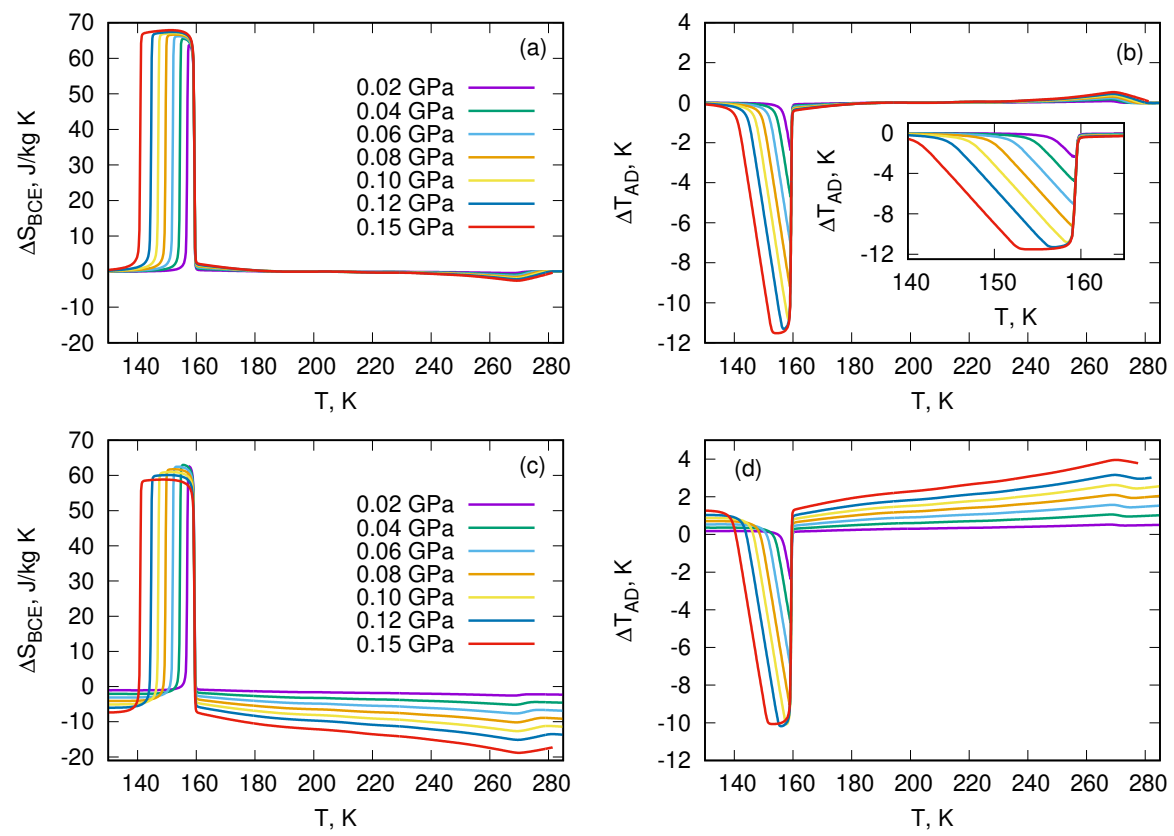

Figure 4: (a) Barocaloric entropy and (b) adiabatic temperature changes at different hydrostatic pressure in a wide temperature range determined without taking into account the effect of thermal expansion of the crystal lattice. Effect of thermal expansion of the crystal lattice on (c) $\triangle S_{B C E}$ and (d) $\Delta T_{A D}$

on $\mathrm{BCE}$ in $\mathrm{NH}_{4} \mathrm{HSO}_{4}$ was studied. This contribution can be evaluated using Maxwell relation $\left(\partial S_{L A T} / \partial p\right)_{T}=-(\partial V / \partial T)_{p}$

$$
\Delta S_{L A T}(T, p)=-\int_{0}^{p}(\partial V / \partial T)_{p} d p \approx-V_{m} \beta_{L A T}(T) p .
$$

Here $\left.V_{m}=6.17 \times 10^{-5} \mathrm{~m}^{3} / \mathrm{mol}\right)$ is the molar volume.

Taken into account the data of the thermal expansion study of the related $\left(\mathrm{NH}_{4}\right)_{2} \mathrm{SO}_{4}$ [15, it was suggested that the values of $\beta_{L A T}$ and $V_{m}$ of $\mathrm{NH}_{4} \mathrm{HSO}_{4}$ are also weakly depend on the pressure. Lattice contribution was determined from the results of dilatometric study of $\mathrm{NH}_{4} \mathrm{HSO}_{4}$ [19].

Fig. 4(c) and (d) demonstrate that due to the same sign of both derivatives, $(\partial V / \partial T)_{T 1}$ and $\left(\partial V_{L A T} / \partial T\right)$, there is a strong increase in $\mathrm{BCE}_{c o n v}$. At $p=0.15 \mathrm{GPa}$, the values $\left(\Delta S_{B C E}\right)_{T 1}=-18.8 \pm 1.5 \mathrm{~J} / \mathrm{kg} \cdot \mathrm{K}$ and $\left(\Delta T_{A D}\right)_{T 1}=$ $4.0 \pm 0.2 \mathrm{~K}$ are about one and a half times higher than even the maximum 
Thus, contributions of $\left(\Delta S_{B C E}^{L A T}\right)_{T 1}$ and $\left(\Delta T_{A D}^{L A T}\right)_{T 1}$ to the full BCE at $T_{1}$ are predominant.

On the other hand, at the same pressure, $\mathrm{BCE}_{i n v}$ associated with the phase transition $P c-P 1\left((\partial V / \partial T)_{T 2}<0\right)$, is reduced to the magnitudes $\left(\Delta S_{B C E}\right)_{T 2}=$ $59 \pm 5 \mathrm{~J} / \mathrm{kg} \cdot \mathrm{K},\left(\Delta T_{A D}\right)_{T 2}=-10.0 \pm 0.8 \mathrm{~K}$ by $\mathrm{BCE}_{\text {conv }}^{L A T}$ arising in accordance with Eq. 2. $\left(\partial V_{L A T} / \partial T\right)>0:\left(\Delta S_{B C E}^{L A T}\right)_{T 2}=-9.0 \pm 0.7 \mathrm{~J} / \mathrm{kg} \cdot \mathrm{K},\left(\Delta T_{A D}^{L A T}\right)_{T 2}=$ $1.5 \pm 0.1 \mathrm{~K}$.

At last, at the third stage, we determine the behavior of extensive and intensive $\mathrm{BCE}$ at $T_{2}$ taken into account the peculiarities of experiments with DTA under pressure. As it was discussed above, increase in pressure strongly decreases the entropy jump $\delta S_{2}$ at the first order phase transition $P c-P 1$ (Fig. 2(b) and (d)), while the total entropy change $\Delta S_{2}$ remains constant. Analyzing the dependencies $S(T, p)$ using both the DTA data under pressure and the effect of the lattice expansion, barocaloric parameters at $T_{2}$ are determined and One can see that in this case the magnitudes of $\left(\Delta S_{B C E}^{\max }\right)_{T 2}$ and $\left(\Delta T_{A D}^{\max }\right)_{T 2}$ are realized at almost the same low pressure which was determined in the second stage (Fig. 4(c) and (d)). The main difference was observed in the form of peaks $\left(\Delta S_{B C E}\right)_{T 2}(T)$ and $\left(\Delta T_{A D}\right)_{T 2}(T)$ which is due to peculiarities of the processes of measuring the heat capacity by methods of adiabatic calorimeter and DTA.

Returning to the results of the BCE study at $T_{1}$, one can confidently argue that it would be interesting and useful to investigate the influence of the thermal expansion of the crystal lattice on conventional BCE in ferroelectrics characterized by large $\beta_{L A T}$ and undergoing order-disorder transformation with

\section{Conclusion}

This paper demonstrates $\mathrm{BCE}$ in $\mathrm{NH}_{4} \mathrm{HSO}_{4}$ undergoing two successive ferroelectric phase transitions characterized by significantly different entropy changes 

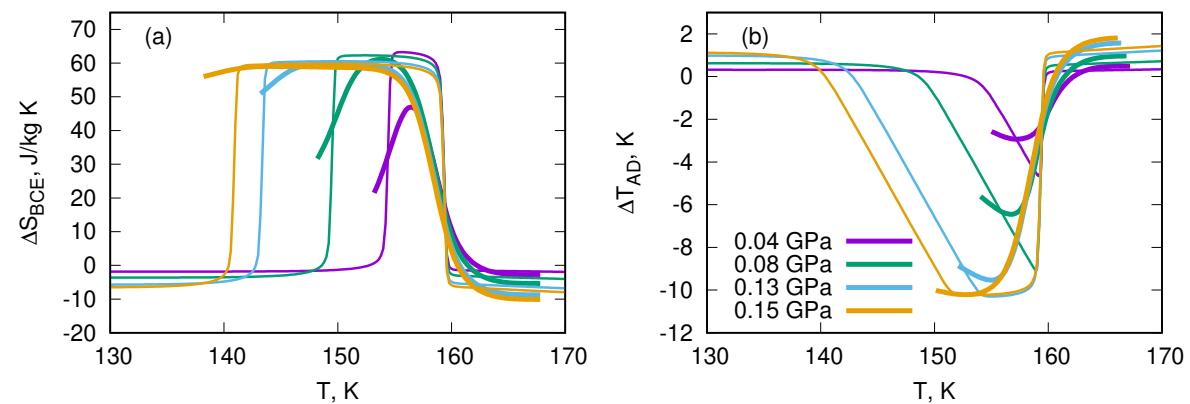

Figure 5: (a) Barocaloric entropy and (b) adiabatic temperature changes at different hydrostatic pressure determined using DTA data on a jump of the entropy (thick lines) and data presented in Fig, 4(c) and (d) (thin lines).

$\left(\Delta S_{1}=1.2 \mathrm{~J} / \mathrm{mol} \cdot \mathrm{K}, \Delta S_{2}=7.6 \mathrm{~J} / \mathrm{mol} \cdot \mathrm{K}\right)$ and large baric coefficients of different sign $\left(d T_{1} / d p=+90 \mathrm{~K} / \mathrm{GPa}\right.$ and $\left.d T_{2} / d p=-123 \mathrm{~K} / \mathrm{GPa}\right)$. Hydrostatic pressure strongly decreases the entropy jump at $T_{2}$ which reaches zero at a pressure of $0.17 \mathrm{GPa}$. Very low pressure is needed to realize the maximum values of the extensive and intensive inverse BCE. Large thermal expansion of the crystal lattice leads to two very important points. Firstly, the conventional BCE can be greatly increased to values much higher than the magnitudes, corresponding to the entropy of the phase transition. Secondly, in the case of the negative baric coefficient, a conversion from the inverse to conventional BCE can be realized by low pressure in the narrow temperature range.

\section{References}

\section{References}

[1] K. A. GschneidnerJr, V. K. Pecharsky, A. O. Tsokol, Recent developments in magnetocaloric materials, Rep. Prog. Phys. 68 (6) (2005) 1479-1539 (2005). doi:10.1088/0034-4885/68/6/r04.

[2] M. Valant, Electrocaloric materials for future solid-state refrigeration tech185 nologies, Prog. Mater. Sci. 57 (6) (2012) 980 - 1009 (2012). doi:10.1016/
j.pmatsci.2012.02.001. 
[3] X. Moya, S. Kar-Narayan, N. D. Mathur, Caloric materials near ferroic phase transitions, Nat. Mater. 13 (2014) 439-450 (2014). doi:10.1038/ nmat3951.

[4] H. Khassaf, T. Patel, R. J. Hebert, S. P. Alpay, Flexocaloric response of epitaxial ferroelectric films, J. Appl. Phys. 123 (2) (2018) 024102 (2018). doi:10.1063/1.5009121.

[5] U. Tomc, J. Tušek, A. Kitanovski, A. Poredoš, A new magnetocaloric refrigeration principle with solid-state thermoelectric thermal diodes, Appl. Thermal Engineering 58 (1) (2013) 1-10 (2013). doi:10.1016/j. applthermaleng.2013.03.063.

[6] U. Plaznik, M. Vrabelj, Z. Kutnjak, B. Malič, A. Poredoš, A. Kitanovski, Electrocaloric cooling: The importance of electric-energy recovery and heat regeneration, EPL (Europhysics Letters) 111 (5) (2015) 57009 (2015). doi: 10.1209/0295-5075/111/57009.

[7] A. Kitanovski, U. Plaznik, U. Tomc, A. Poredoš, Present and future caloric refrigeration and heat-pump technologies, Int. J. Refrigeration 57 (2015) 288 - 298 (2015). doi:10.1016/j.ijrefrig.2015.06.008

[8] N. Michaelis, F. Welsch, S.-M. Kirsch, M. Schmidt, S. Seelecke, A. Schütze, Experimental parameter identification for elastocaloric air cooling, Int. J. Refrigeration 100 (2019) 167 - 174 (2019). doi:10.1016/j.ijrefrig. 2019.01 .006

[9] M. Gorev, E. Bogdanov, I. Flerov, T-p phase diagrams and the barocaloric effect in materials with successive phase transitions, J. Phys. D: Appl. Phys. 50 (38) (2017) 384002 (2017). doi:10.1088/1361-6463/aa8025.

[10] M. Gorev, E. Bogdanov, I. Flerov, Conventional and inverse barocaloric effects around triple points in ferroelastics $\left(\mathrm{NH}_{4}\right)_{3} \mathrm{NbOF}_{6}$ and $\left(\mathrm{NH}_{4}\right)_{3} \mathrm{TiOF}_{5}$,

口 Scripta Materialia 139 (2017) 53-57 (2017). doi:10.1016/j.scriptamat. 2017.06 .022 
[11] E. Mikhaleva, I. Flerov, A. Kartashev, M. Gorev, A. Cherepakhin, K. Sablina, N. Mikhashenok, N. Volkov, A. Shabanov, Caloric effects and phase transitions in ferromagnetic-ferroelectric composites $\mathrm{xLa}_{0} \cdot{ }_{7} \mathrm{~Pb}_{0}$. ${ }_{3} \mathrm{MnO}_{3}-(1-\mathrm{x}) \mathrm{PbTiO}_{3}$, J. Mater. Res. 28 (24) (2013) 3322-3331 (2013). doi:10.1557/jmr.2013.360

[12] K. Alex Müller, F. Fauth, S. Fischer, M. Koch, A. Furrer, P. Lacorre, Cooling by adiabatic pressure application in $\mathrm{Pr}_{1}-\mathrm{xLaxNiO}_{3}$, Appl. Phys. Letters 73 (8) (1998) 1056-1058 (1998). doi:10.1063/1.122083.

[13] T. Strässle, A. Furrer, Z. Hossain, C. Geibel, Magnetic cooling by the application of external pressure in rare-earth compounds, Phys. Rev. B 67 (2003) 054407 (2003). doi:10.1103/PhysRevB.67.054407

[14] N. A. de Oliveira, Barocaloric effect and the pressure induced solid state 口 refrigerator, J. Appl. Phys. 109 (5) (2011) 053515 (2011). doi:10.1063/ 1.3556740 .

[15] P. Lloveras, E. Stern-Taulats, M. Barrio, J.-L. Tamarit, S. Crossley, W. Li, V. Pomjakushin, A. Planes, L. Manosa, N. D. Mathur, X. Moya, Giant barocaloric effects at low pressure in ferrielectric ammonium sulphate, Nat. Commun. 6 (2015) 8801 (2015). doi:10.1038/ncomms9801.

[16] H. Khassaf, T. Patel, S. P. Alpay, Combined intrinsic elastocaloric and electrocaloric properties of ferroelectrics, J. Appl. Phys. 121 (14) (2017) 144102 (2017). doi:10.1063/1.4980098.

[17] Y. Liu, J. Wei, P.-E. Janolin, I. C. Infante, X. Lou, B. Dkhil, Giant roomtemperature barocaloric effect and pressure-mediated electrocaloric effect in $\mathrm{BaTiO}_{3}$ single crystal, Appl. Phys. Letters 104 (16) (2014) 162904 (2014). doi:10.1063/1.4873162.

[18] M. V. Gorev, I. N. Flerov, E. V. Bogdanov, V. N. Voronov, N. M. Laptash, Barocaloric effect near the structural phase transition in the $\mathrm{Rb}_{2} \mathrm{KTiOF}_{5}$ 
oxyfluoride, Phys. Solid State 52 (2) (2010) 377-383 (2010). doi:10.1134/ S1063783410020253.

[19] E. Mikhaleva, I. Flerov, A. Kartashev, M. Gorev, E. Bogdanov, V. Bondarev, Thermal, dielectric and barocaloric properties of $\mathrm{NH}_{4} \mathrm{HSO}_{4}$ crystallized from an aqueous solution and the melt, Solid State Sciences 67 (2017) 1-7 (2017). doi:10.1016/j.solidstatesciences.2017.03.004.

[20] R. Pepinsky, K. Vedam, S. Hoshino, Y. Okaya, Ammonium hydrogen sulfate: A new ferroelectric with low coercive field, Phys. Rev. 111 (1958) 1508-1510 (1958). doi:10.1103/PhysRev.111.1508.

[21] D. Swain, V. S. Bhadram, P. Chowdhury, C. Narayana, Raman and x-ray investigations of ferroelectric phase transition in $\mathrm{NH}_{4} \mathrm{HSO}_{4}$, J. Phys. Chem. A 116 (2012) 223-230 (2012). doi:10.1021/jp2075868.

[22] R. Pirc, Z. Kutnjak, R. Blinc, Q. M. Zhang, Upper bounds on the electrocaloric effect in polar solids, Appl. Phys. Letters 98 (2) (2011) 021909 (2011). doi:10.1063/1.3543628. 

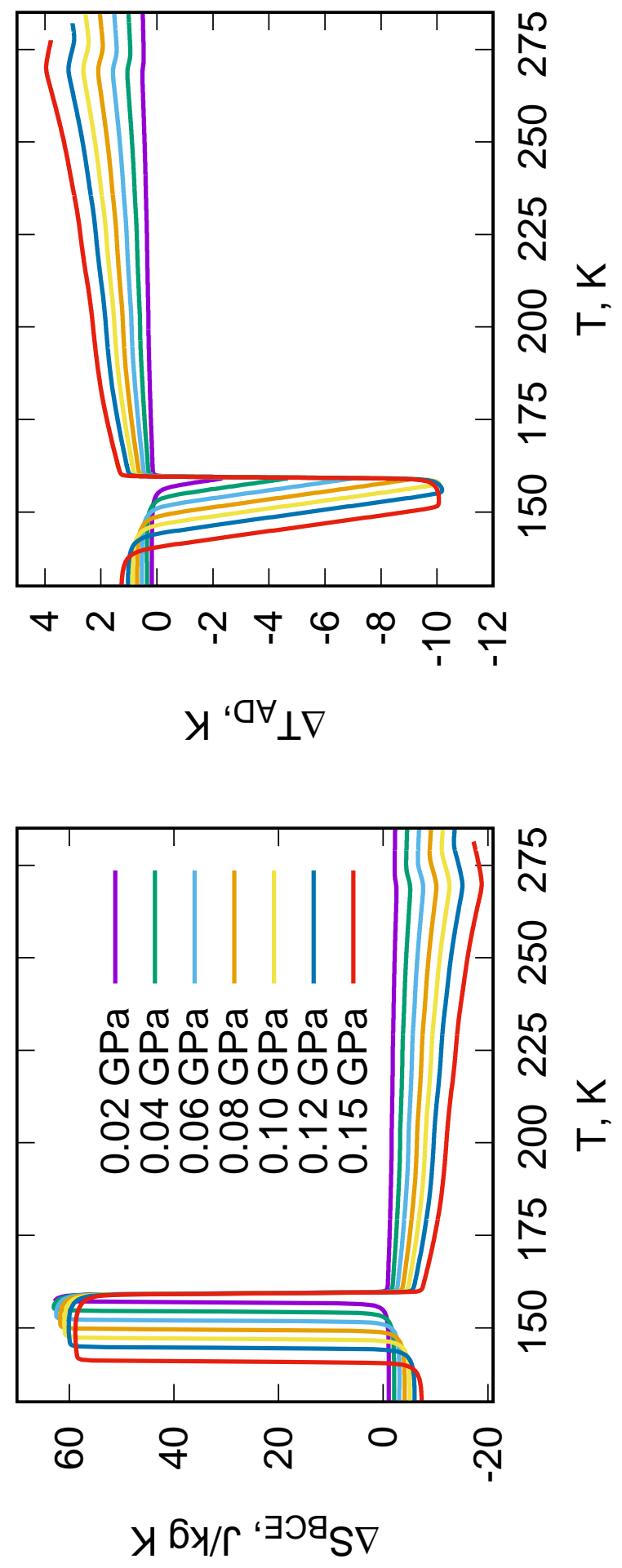
- Temperature/entropy - pressure phase diagrams of ferroelectric $\mathrm{NH}_{4} \mathrm{HSO}_{4}$ were studied

- Pressure strongly effects on the temperature and entropy of the phase transitions.

- Low pressure induces large inverse barocaloric effect at first order transformation.

- Expansion of the crystal lattice strongly affects the barocaloric efficiency.

- The conversion between the inverse and conventional barocaloric effects is found. 
LaTeX Source Files
Click here to download LaTeX Source Files: manuscript_rev.zip LaTeX Source Files
Click here to download LaTeX Source Files: manuscript_rev.zip Click here to download LaTeX Source Files: manuscript revizip

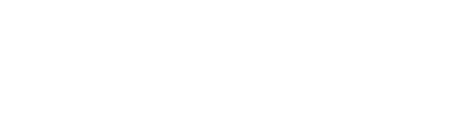

$\sqrt{2}$

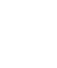

$\sqrt{2}$

(1) (1)

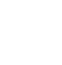
(1) (1) (1) . . . . . . . . . . 
LaTeX Source Files
Click here to download LaTeX Source Files: manuscript_BCE_AHS.zip

LaTeX Source Files
Click here to download LaTeX Source Files: manuscript_BCE_AHS.zip Click here to download LaTeX Source Files: manuscript_BCE_AHS.zip

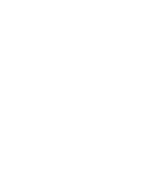

(1)

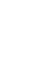
(1) (1) (n) (1) (1) (1) (n) . .

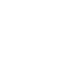

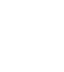

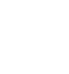

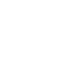

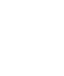

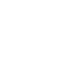

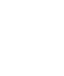

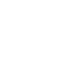

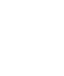

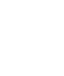

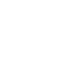

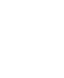

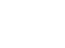


LaTeX Source Files
Click here to download LaTeX Source Files: manuscript_rev2 (1).zip

LaTeX Source Files
Click here to download LaTeX Source Files: manuscript_rev2 (1).zip

LaTeX Source Files
Click here to download LaTeX Source Files: manuscript_rev2 (1).zip

(1)

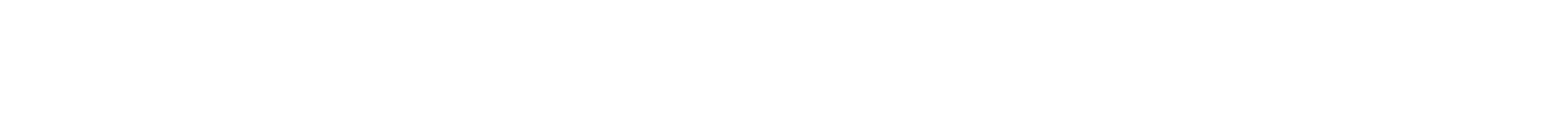

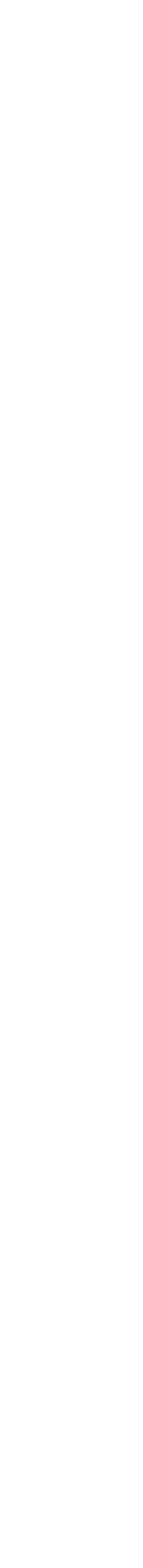

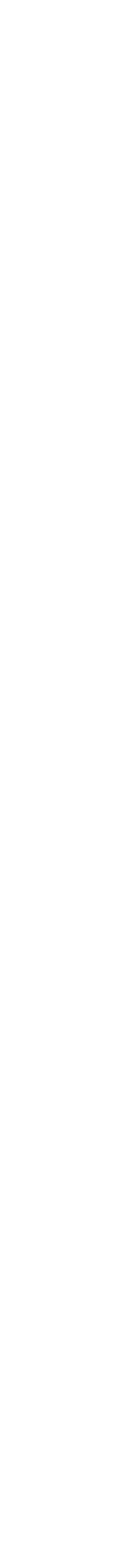

